\title{
Dynamical analysis of a reduced model for the North Atlantic Oscillation
}

\author{
Courtney Quinn*, Dylan Harries, and Terence J. O’Kane \\ CSIRO Oceans and Atmosphere, Hobart, Tasmania, Australia
}

This manuscript has been peer-reviewed and accepted for publication in Journal of the

${ }_{5}$ Atmospheric Sciences. Once published, the final version of this manuscript will be available via

the 'Peer-reviewed Publication DOI' link. Please feel free to contact the authors with any feedback.

${ }_{8}^{*}$ Corresponding author: Courtney Quinn, courtney.quinn@ csiro.au 


\begin{abstract}
The dynamics of the North Atlantic Oscillation (NAO) are analyzed through a data-driven model obtained from atmospheric reanalysis data. We apply a regularized vector autoregressive clustering technique to identify recurrent and persistent states of atmospheric circulation patterns in the North Atlantic sector $\left(110^{\circ} \mathrm{W}-0^{\circ} \mathrm{E}, 20^{\circ} \mathrm{N}-90^{\circ} \mathrm{N}\right)$. In order to analyze the dynamics associated with the resulting cluster-based models, we define a time-dependent linear delayed map with a switching sequence set a priori by the cluster affiliations at each time step. Using a method for computing the covariant Lyapunov vectors (CLVs) over various time windows, we produce sets of mixed singular vectors (for short windows) and approximate the asymptotic CLVs (for longer windows). The growth rates and alignment of the resulting time-dependent vectors are then analyzed. We find that the window chosen to compute the vectors acts as a filter on the dynamics. For short windows, the alignment and changes in growth rates are indicative of individual transitions between persistent states. For long windows, we observe an emergent annual signal manifest in the alignment of the CLVs characteristic of the observed seasonality in the NAO index. Analysis of the average finitetime dimension reveals the $\mathrm{NAO}^{-}$as the most unstable state relative to the $\mathrm{NAO}^{+}$, with persistent AR states largely stable. Our results agree with other recent theoretical and empirical studies that have shown blocking events to have less predictability than periods of enhanced zonal flow.
\end{abstract}




\section{Introduction}

The North Atlantic Oscillation (NAO) is a prominent mode of variability in the Northern Hemisphere $(\mathrm{NH})$ atmospheric circulation. Concentrated between the eastern North American and western European continent, the oscillation characterizes the behavior of large regions of high and low pressure anomalies over the North Atlantic Ocean. While the background state of atmospheric pressure in this region consists of lower pressure to the north and higher pressure in the mid-latitudes, the NAO describes the modulation to this background state, either enhancing it (positive phase) or weakening it (negative phase). The changes to the background state of atmospheric pressure over the Atlantic affect wind speed and direction, heat and moisture transport, and storm numbers and intensity (Hurrell et al. 2013). The instabilities driving transitions between the phases can develop rapidly and are therefore difficult to predict. This leads to impacts across many socioeconomic sectors, and therefore motivates further study into the dynamics associated with such a phenomenon.

The two phases of the NAO and their respective associated pressure differences have opposing effects on the observed atmospheric physics. The positive phase enhances the zonal flow across the North Atlantic Ocean with much stronger than average westerlies in the mid-latitudes (Visbeck et al. 2001). These westerlies bring warmer weather to the European continent, particularly in the winter, as well as stronger and more frequent storms to northern Europe (drier conditions in southern Europe) (Hurrell 1995). In contrast, the negative phase weakens the mid-latitude westerlies and is associated with increased blocking events in the North Atlantic region (Shabbar et al. 2001; Benedict et al. 2004; Croci-Maspoli et al. 2007; Woollings et al. 2008) and anomalously cold temperatures over the eastern North American and northern European continents (Shabbar et al. 2001). Although the NAO has variability on interannual and decadal timescales (Hurrell 
1995; Stephenson et al. 2000), the complicated relationship of the individual NAO phases to synoptic scale variability makes it a complex phenomenon to study dynamically.

An important contributor to the NAO is the interplay between barotropic and baroclinic instability. Some of the simpler conceptual models proposed for the observed variability of the NAO include nonlinear barotropic models forced either by a random process imitating baroclinic instability (Vallis et al. 2004) or a synoptic-scale wave-maker function (Luo et al. 2007a,b,c; Luo and Cha 2012). In the former case, the dipole structure in the pressure field is a result of a dipolar circulation anomaly caused by the large-scale vorticity stirring in the Atlantic storm track (Vallis et al. 2004). The latter case emphasizes the importance of a preexisting dipole planetary-scale wave whose spatial structure must match that of the synoptic-scale wave forcing (Luo et al. 2007a), and it is shown in such a model that wave-breaking is not a necessary condition for NAO events to occur (Luo et al. 2007c). When a variable Atlantic mean westerly wind is included in the model, it can also induce direct transitions between phases (Luo and Cha 2012). There has also been a considerable amount of work into identifying the dynamical drivers of the NAO through analyzing the output of general circulation models (GCMs). Feldstein (2003) found that initiation of a positive phase resulted from anomalous wavetrain propagation, while the negative phase resulted from in situ growth of the NAO anomaly itself. Other studies have confirmed the necessity of wave-breaking for the initiation of both phases, with anticyclonic (cyclonic) wave-breaking leading to a positive (negative) phase (Benedict et al. 2004; Franzke et al. 2004). Franzke et al. (2004) also conclude that the latitudinal positioning of the Pacific storm track aids in the determination of the phase. Much work has shown the Madden-Julian oscillation (MJO) is strongly connected to the phase of the NAO (Frederiksen and Frederiksen 1993; Cassou 2008; Frederiksen and Lin 2013; Lin et al. 2018). Cassou (2008) found that when the MJO initiates a Rossby wave disturbance in the western-central tropical Pacific, a positive NAO event was found to occur, whereas negative 
NAO events resulted from eastern-tropical Pacific or western Atlantic disturbances that modified the North Atlantic storm track. The MJO-NAO teleconnection can be shown to largely fall within the general theory for intraseasonal oscillations first proposed by Frederiksen (2002).

It is clear from the discussion of the above studies that much remains to be explained regarding the dynamics governing observed transitions between, and persistence of, the respective NAO phases and relationship to the associated mid-latitude (Atlantic Ridge, Scandinavian blocking etc), tropical (MJO), and polar (Arctic Oscillation) teleconnections. One approach that has been suggested to characterize the instabilities governing changes in atmospheric flow patterns is through the study of covariant Lyapunov vectors (CLVs). These vectors give a basis on the tangent linear space and provide directions in phase space of linear perturbations to a nonlinear background flow (Ruelle 1979; Trevisan and Pancotti 1998; Ginelli et al. 2007; Wolfe and Samelson 2007; Kuptsov and Parlitz 2012). Schubert and Lucarini $(2015,2016)$ first applied this method to a two-layer quasigeostrophic barotropic-baroclinic channel model employing the calculated CLVs to characterize the stability of, and transitions between, respective zonal and blocked states and to explain the variance of the modelled atmospheric dynamics. They found that the unstable CLVs showed enhanced instability during blocked events, where the contributing process to the enhancement of instability depended on the baroclinicity of the background flow. In a move towards using more realistic representations of the dynamics, recent studies have employed finite-time dynamical properties (such as finite-time growth rates of the CLVs or the instantaneous attractor dimension) to characterize the NAO behavior. The increasing finite-time instability during blocking events associated with the negative NAO phase was seen in a three-layer quasi-geostrophic model in spherical geometry (Lucarini and Gritsun 2020), as well as in reanalysis data (Faranda et al. 2017). This apparent contradiction between the greater than average instability and the expected enhanced predictability during a persistent blocked flow was suggested to be related to the difficulty in 
predicting block onset and decay; the formation and decay of a block was found to be associated with the largest increases in the dimension of the unstable manifold (Lucarini and Gritsun 2020). An additional way to study the dynamics of the observed NAO is through the analysis of datadriven models that identify the teleconnection in high dimensional raw observed or simulated data. Starting from the premise that atmospheric flows exhibit a set of weather regimes (Legras and Ghil 1985; Vautard 1990; Kimoto and Ghil 1993a), clustering methods (e.g., Mo and Ghil 1988; Stone 1989; Molteni et al. 1990; Hannachi and Legras 1995; Kidson 2000; Renwick 2005; Straus et al. 2007; Stan and Straus 2007; Fereday et al. 2008; Huth et al. 2008; Pohl and Fauchereau 2012; Neal et al. 2016) generally detect patterns associated with recurrent behavior or slow evolution of the system with respect to a reference time-scale. When applied to the circulation over the North Atlantic (see, e.g., Vautard 1990; Cheng and Wallace 1993; Michelangeli et al. 1995; Smyth et al. 1999; Cassou et al. 2005; Cassou 2008), a small number of regimes are identified and may be associated with the NAO as well as preferred blocking patterns. On the other hand, the simplest clustering-based methods do not explicitly incorporate dynamical information (Harries and O' Kane 2020), which must be studied using various post hoc approaches (Vautard 1990; Kimoto and Ghil 1993b; Crommelin 2004; Fereday 2017).

Latent variable models, such as hidden Markov models (HMMs) and other state space models (e.g., Majda et al. 2006; Franzke et al. 2008, 2011), attempt to better account for these important dynamical aspects. HMM studies of the North Atlantic circulation have been shown to identify persistent hidden regimes corresponding to the NAO and East Atlantic pattern (Franzke et al. 2011) and used to study signals relating to regime transitions (Franzke et al. 2011; Tantet et al. 2015). However, the assumption that the flow is well-described by a time-homogeneous Markov chain need not be satisfied in practice, nor are the extracted regimes necessarily metastable. 
One such approach that has recently been found to be effective in extracting metastable regimes states makes use of the so-called finite element clustering with bounded variation (FEM-BV) framework (Horenko 2009, 2010a,b; Metzner et al. 2012). As in an HMM, the FEM-BV method presumes the existence of a finite number of hidden states, each having time-independent properties, and a switching process describing transitions between the states. This switching process is not required to be governed by a Markov chain; instead, the model is regularized to enforce some level of persistent residence in the states. The system is thus described in terms of a set of locally stationary states, e.g., in the FEM-BV-VAR method, by locally stationary linear vector autoregressive (VAR) processes. In applications to the mid-latitude troposphere (Franzke et al. 2009; O'Kane et al. 2013b; Franzke et al. 2015; Risbey et al. 2015; O’Kane et al. 2016, 2017; Falkena et al. 2020) and large-scale ocean circulation (O'Kane et al. 2013a), the FEM-BV-VAR method and its variants have been found to identify persistent states that can be identified as large-scale coherent structures. Additional applications of the FEM-BV-VAR method include studies of the atmospheric boundary layer (Vercauteren and Klein 2015; Vercauteren et al. 2016).

The above studies have demonstrated that the FEM-BV-VAR method extracts reasonable metastable states. The associated switching sequences, on the other hand, have received less attention, with most focus given to investigating multiyear trends in the occurrence of states (O'Kane et al. 2016, and references therein) and their association with extremes (Risbey et al. 2018). At shorter time-scales, it might be hoped that the state transition sequence captures at least some aspects of the dynamics associated with regime transitions, in spite of the severe dimension reduction involved in formulating the model. More generally, it is not clear whether dynamical signals such as the increase in finite-time dimension during blocking events that is seen in both theoretical models and the data are also captured by the widely-used data-driven models. In this study, we investigate these questions in the context of a model for the NAO derived from 
an FEM-BV-VAR cluster analysis. When applied to the atmospheric circulation in the Atlantic sector, the FEM-BV-VAR method yields a set of states consistent with differing phases of the NAO. By treating the clustering as a discrete linear delay system, it is possible to directly compute the Lyapunov spectrum and CLVs of the model, as well as dynamical indicators of transitions such as increased finite-time instability (Norwood et al. 2013) and alignment of CLVs (Beims and Gallas 2016; Sharafi et al. 2017; Kuptsov and Kuznetsov 2018). The relationship between these dynamical quantities and the particular regime transitions can then be compared to assess whether the reduced-order model exhibits non-trivial dynamics.

In this study we analyze the optimal model for the NAO resulting from applying the FEMBV-VAR method to atmospheric reanalysis data. The remainder of this article is structured as follows. In section 2 the data and clustering methods used to derive a reduced order model for circulation regimes is described. We introduce the general properties of the optimal model and validate it against an observed NAO index. In section 3 we define the corresponding discrete time dynamical system through construction of a delay-embedded linear map that corresponds to the time-dependent dynamics of the optimal model from the fit. Through this novel interpretation of the system we calculate the corresponding CLVs and their properties as they evolve in time. We focus on the characterization of persistent states and analyze how the dynamical properties relate to the transitioning behavior of the model, both on short and long time-scales. Finally, in section 4 we summarize our findings. 


\section{Identifying North Atlantic circulation regimes}

\section{a. Data}

We examine the $\mathrm{NH}$ mid-tropospheric circulation in terms of daily mean $500 \mathrm{hPa}$ geopotential height $\left(Z_{g 500 \mathrm{hPa}}\right)$ fields obtained from the National Centers for Environmental Prediction/National Center for Atmospheric Research (NCEP/NCAR) Reanalysis 1 (Kalnay et al. 1996).

The NCEP/NCAR Reanalysis 1 (NNR1) atmospheric reanalysis spans 1948 to present with a T62 resolution on 28 vertical levels and is constrained by both surface and atmospheric observational data. The $Z_{g 500 \mathrm{hPa}}$ data are provided on a global $2.5^{\circ} \times 2.5^{\circ}$ latitude-longitude grid, from which we compute daily height anomalies, $Z_{g 500 \mathrm{hPa}}^{\prime}$, by subtracting the daily climatological mean determined from the 1 January 1979 to 31 December 2018 reference period. An initial dimension reduction is carried out by performing an EOF analysis of the latitude-weighted daily height anomalies in the North Atlantic sector $\left(110^{\circ} \mathrm{W}-0^{\circ} \mathrm{E}, 20^{\circ} \mathrm{N}-90^{\circ} \mathrm{N}\right)$ between 1 January 1979 and 31 December 2018, including all seasons. This preprocessing step is required to reduce the overall dimensionality of the data in order to render the subsequent clustering analysis, now applied to the retained principal components (PCs) rather than the full gridded fields, tractable. Otherwise, no further use is made of the corresponding spatial patterns in defining the extracted regimes. The number of PCs retained should be large enough to capture the relevant dynamics driving the processes of interest, while at the same time not being so large that the clustering problem is ill-posed. In carrying out sensitivity analyses with respect to the number of retained PCs, it was found that $d=10 \mathrm{PCs}$ was insufficient to capture the meridionally oriented dipolar structures associated with the NAO, with the reduced order model states instead tending to consist of predominantly zonally oriented wavetrains, as previously observed in O'Kane et al. (2017). For $d=20$ PCs, on the other hand, we find that the expected structures are found in the reduced order model, as discussed below. In the following we 
therefore choose to keep the leading $d=20 \mathrm{PCs}$, accounting for approximately $91 \%$ of the total variance; the corresponding EOFs are shown in appendix A. Additionally, to assess the qualitative behavior of the regimes identified by the clustering analysis, we make use of the daily NAO index ${ }^{1}$ provided by the National Oceanic and Atmospheric Administration Climate Prediction Center (NOAA CPC) (Barnston and Livezey 1987).

\section{b. FEM-BV-VAR clustering}

Given the daily timeseries of $d=20$ PCs between 1 January 1979 and 31 December 2018, corresponding to a sample of length $T=14610$ days, we next extract a set of persistent states by applying the FEM-BV-VAR clustering method (Horenko 2010b; Metzner et al. 2012).

In this approach, the behavior of the system is taken to be described by an underlying model determined by a set of generally time-dependent parameters $\Theta(t)$. Specifically, in the FEM-BVVAR case, the stochastic model is taken to be of the form

$$
\mathbf{x}_{t}=\boldsymbol{\mu}(t)+\sum_{\tau=1}^{m} \mathbf{A}_{\tau}(t) \mathbf{x}_{t-\tau}+\boldsymbol{\epsilon}_{t}
$$

where $\Theta(t)=\left(\mu_{t}, \mathbf{A}_{1}(t), \ldots, \mathbf{A}_{m}(t), \boldsymbol{\Sigma}(t)\right)$ is a vector of time-dependent model parameters for an order $m$ linear autoregressive model with mean vector $\boldsymbol{\mu}(t)$ and random noise $\epsilon_{t}$ with time-varying covariance matrix $\Sigma(t)$. To arrive at a well-posed problem for estimating the model parameters, it is then assumed that the full, non-stationary system can be well approximated in terms of transitions between a finite set of $K$ states. These states are assumed to be individually stationary and determined by a set of fixed, time-independent parameters $\Theta_{i}, i=1, \ldots, K$, i.e., the system is assumed to be locally stationary (Metzner et al. 2012). The original time-dependence of the model parameters then arises via the switching of the system between states. The time-scales associated with the individual states and with the underlying switching process may in general differ, making

${ }^{1}$ https://www.cpc.ncep.noaa.gov/products/precip/CWlink/pna/nao.shtml 
the method suitable for analyzing the multiscale dynamics typical of the atmospheric circulation. The resulting model is interpreted as representing the observed fields in terms of a set of recurrent circulation regimes that govern the local, short-term (e.g., day-to-day) variability, which the system repeatedly transitions between.

To determine both an assignment of individual days to a state as well as the parameters $\Theta_{i}$ characterizing each state, we minimize a loss function of the form

$$
L(\boldsymbol{\Theta}, \boldsymbol{\Gamma})=\frac{1}{T} \sum_{t=1}^{T} \sum_{i=1}^{K}\left[\gamma_{t}\right]_{i} \ell_{i}\left(\mathbf{x}_{t}, \Theta_{i}\right),
$$

where $\mathbf{x}_{t} \in \mathbb{R}^{d}$ denotes the vector of PCs at time $t, \boldsymbol{\Theta}=\left(\Theta_{1}, \ldots, \Theta_{K}\right)$ denotes the combined set of parameters for all states, and the functions $\ell_{i}\left(\mathbf{x}_{t}, \Theta_{i}\right)$ are appropriately chosen loss functions for each of the $K$ states quantifying the level of fit under that state for given $\Theta_{i}$, e.g., the squared error or negative log-likelihood. The sequence of state assignments is encoded by the state affiliations $\gamma_{t} \in \mathbb{R}^{K}$. At a given time $t$, these affiliations are required to satisfy

$$
\sum_{i=1}^{K}\left[\gamma_{t}\right]_{i}=1, \quad\left[\gamma_{t}\right]_{i} \geq 0 \quad \forall i=1, \ldots, K,
$$

such that the loss function is a convex combination of the individual losses and the complete set of affiliations $\boldsymbol{\Gamma}^{T}=\left[\gamma_{1}^{T}, \ldots, \gamma_{T}^{T}\right] \in \mathbb{R}^{K \times T}$ may be interpreted as providing a soft clustering of the data into the $K$ states. The observed persistence of large-scale coherent features in the mid-latitude troposphere implies that the switching process described by the affiliations $\Gamma$ should also exhibit some degree of persistence, yielding regimes that are metastable. To enforce this behavior, the affiliation sequence is required to satisfy a constraint on the total variation norm of the sequence ${ }^{2}$,

${ }^{2}$ In the usual formulation of FEM-BV clustering, it is further assumed that the affiliations can be expressed in terms of a set of compactly supported basis functions. When each basis function is non-zero over more than one time step, this essentially imposes a minimum length of time that must be spent in a given state. We choose triangular basis functions that are non-vanishing at only a single time point, allowing state transitions between adjacent time points. 
of the form

$$
\sum_{t=1}^{T-1}\left|\left[\gamma_{t+1}\right]_{i}-\left[\gamma_{t}\right]_{i}\right| \leq C_{T}, \quad \forall i=1, \ldots, K,
$$

for some constant $C_{T}$. Each term in this sum is non-zero only if the affiliations differ between times $t$ and $t+1$, corresponding to a transition between states, so that this constraint imposes an upper bound on the total number of transitions between states. It is more convenient to express this constraint in terms of a "typical" state length $p \geq 0$ that is independent of the time series length, in terms of which we define $C_{T}$ as

$$
C_{T}=\frac{T}{p}-1
$$

The form of the loss functions $\ell_{i}\left(\mathbf{x}_{t}, \Theta_{i}\right)$ is governed by the assumed dynamics within the hidden states. For the FEM-BV-VAR clustering method, the time evolution of the system within a given state is described by Eq. (1) where $\Theta(t)$ is replaced by $\Theta_{i}=\left(\boldsymbol{\mu}^{(i)}, \mathbf{A}_{1}^{(i)}, \ldots, \mathbf{A}_{m}^{(i)}, \mathbf{\Sigma}^{(i)}\right)$ for each state $i \in\{1, \ldots, K\}$. For simplicity, we assume the same order $m$ for all $K$ states; moreover, we assume that some number $m_{\max } \geq m$ of samples are held-out from the start of the time series to provide the required initial values, leaving $T-m_{\max }$ samples to be modeled. A particular state is then fully specified by the parameters $\Theta_{i}$, and the corresponding loss function is chosen to be the squared residual

$$
\ell_{i}\left(\mathbf{x}_{t}, \Theta_{i}\right)=\left\|\mathbf{x}_{t}-\boldsymbol{\mu}^{(i)}-\sum_{\tau=1}^{m} \mathbf{A}_{\tau}^{(i)} \mathbf{x}_{t-\tau}\right\|^{2} .
$$

A numerical method for finding the minimum of the resulting loss function with respect to $\Theta$ and $\boldsymbol{\Gamma}$ is summarized in appendix B.

The number of clusters $K$, VAR order $m$, and state length $p$ constitute the set of hyperparameters that must be chosen beforehand when applying the above procedure. To determine reasonable choices for these hyperparameters, we perform a grid search over all combinations of $K \in\{1,2,3\}$, $m \in\{0,1,2,3,4,5\}$ days (requiring $m_{\max }=5$ days), and $p \in\{0,5,10, \ldots, 55,60\}$ days. To compare 
models with different hyperparameter settings, we use a rolling origin cross-validation procedure (described in appendix B) to generate estimates of the out-of-sample reconstruction root mean square error (RMSE) for each combination of hyperparameters. Lower values for this measure indicate a reasonable compromise between fitting the data well without overfitting to the training data, and so we select as our optimal model the set of hyperparameters that minimize this metric. The results of this cross-validation procedure, using $N_{\text {fold }}=10$ cross-validation folds, are summarized in FIg. 1. The minimal mean test set reconstruction RMSE is found for $K=3$ states, $m=3$ days, and a typical state length of $p=5$ days. The reconstruction error is, however, rather similar for $K=2$ or $3, m \geq 3$ days, and $p \leq 20$ days, indicating relatively low sensitivity to the choice of persistence so long as the state length is sufficiently short. We note that a typical state length of $\sim 5$ days is consistent with previous results identifying Euro-Atlantic regimes with an FEM-BV variant of $k$-means clustering (Falkena et al. 2020) in which an optimal value of 6.8 days is found based on information criteria applied with a fixed number of $K=4$ clusters.

\section{c. Properties of the optimal model}

Given the fitted affiliation sequence corresponding to the selected model, we assign each time to a state $i_{t} \in\{1,2,3\}$ according to

$$
i_{t}=\underset{j}{\arg \max }\left[\gamma_{t}\right]_{j} .
$$

We do not place a threshold on the number of consecutive days used to define a state, as some level of persistence is already built-in to the clustering model. Composites of the height anomalies assigned to each state in this way are shown in Fig. 2 for the optimal model with $K=3$ states, memory $m=3$ days, and typical state length $p=5$ days. Two states strongly resemble the positive and negative phases of the NAO (Barnston and Livezey 1987), denoted in FIG. 2 by $\mathrm{NAO}^{+}$and $\mathrm{NAO}^{-}$, respectively. The remaining state is somewhat similar to the East Atlantic pattern or 
Atlantic Ridge (AR) pattern (Straus et al. 2017), representing blocking activity in the mid-Atlantic and which has previously been linked to surface temperature extremes in western Europe (Plaut and Simmonet 2001; Cassou et al. 2005). TABLE 1 and TABLE 2 summarize the temporal characteristics of the states in terms of the number of consecutive days spent resident within each state and the frequency of particular transitions. The model has much longer maximum residency lengths in the $\mathrm{NAO}^{-}$state than in the $\mathrm{NAO}^{+}$or the AR states, and generally remains in the $\mathrm{NAO}^{-}$state for longer than either of the other two states. For all three states, the minimum length of time spent in the state is one day, indicating the presence of periods of rapid switching between states. In particular, this implies that fast dynamics, with a time-scale of a day or so, are present in the model in addition to the persistent states. The number of consecutive days spent within a state exhibits a seasonal cycle, with long runs of $\mathrm{NAO}^{-}$states occurring during the boreal summer (JJA) and more equal state lengths during DJF. This is also evident in TABLE 2, which shows a predominance of $\mathrm{NAO}^{-}$ states during JJA and fewer state transitions overall. The $\mathrm{NAO}^{-}$state occurs least frequently during DJF, when most days are assigned to the $\mathrm{AR}$ and $\mathrm{NAO}^{+}$states; the former state is associated in all seasons with a weakening of the mid-latitude zonal flow and in particular with lower maxima in the zonal mean low-level westerlies over the Atlantic, which are more typical of the JJA flow (not shown). Transitioning between states occurs more frequently outside of boreal summer. At the level of particular state transitions, the number of transitions out of the $\mathrm{NAO}^{-}$state is essentially unchanged between DJF and JJA. In JJA, transitions occur preferentially to and from the $\mathrm{NAO}^{-}$ state, while in DJF a larger proportion of transitions are between the $\mathrm{AR}$ and $\mathrm{NAO}^{+}$states.

The state assignments produced by the FEM-BV-VAR fit provide a discrete index measuring the expression of the associated mode on each day. To verify that the occurrence of the NAO-like states shown in FIG. 2 reflects the observed behavior of the NAO, we compare the model affiliation sequence to the NOAA CPC NAO index. As a measure of similarity, we compare the percentage 
of days assigned to the $\mathrm{NAO}^{-}$state with the percentage of days that the $\mathrm{CPC}$ index is negative, defining an $\mathrm{NAO}^{-}$residency percent for both the model and the continuous index. To focus on longer term variability, we compare either the result of computing the residency percent over a one year sliding window, i.e.,

$$
\begin{aligned}
R_{S W}^{\text {model }}(t) & =\sum_{t^{\prime}=t-365}^{t} \frac{\mathbb{I}\left(i_{t^{\prime}}=2\right)}{365}, \\
R_{S W}^{\mathrm{CPC}}(t) & =\sum_{t^{\prime}=t-365}^{t} \frac{\mathbb{I}\left(\mathrm{CPC} \text { index }\left(t^{\prime}\right)<0\right)}{365},
\end{aligned}
$$

where $\mathbb{I}(x)$ is an indicator function equal to one if $x$ is true and zero otherwise, or by applying a LOWESS smoothing (Cleveland 1979) to the fraction of $\mathrm{NAO}^{-}$days in each year. The results of this comparison are shown in FIG. 3. There is a high correlation between the percent of days assigned to the $\mathrm{NAO}^{-}$state in the model and the percent of days with a negative NAO index ( $r \approx 0.74$ between the sliding window time series and $r \approx 0.8$ for the series of annual counts), suggesting that occurrences of the FEM-BV-VAR $\mathrm{NAO}^{-}$state do broadly correspond to conditions characteristic of the negative phase of the NAO. Comparable results were found by Risbey et al. (2015).

\section{Dynamical Analysis}

Based on the above analysis we have some confidence that the optimal FEM-BV-VAR model extracts a set of metastable states that can be related to coherent features in the North Atlantic.

We next assess whether a simplified dynamical model derived from this fit can be used to study the dynamics associated with regime transitions between those states. To do so, the optimal FEMBV-VAR fit with $K=3, m=3$ days, and $p=5$ days can be naturally interpreted as a discrete time 
system based on Eq. (1) in which the time evolution is given by

$$
\mathbf{x}_{t+1}= \begin{cases}\boldsymbol{\mu}^{(1)}+\mathbf{A}_{1}^{(1)} \mathbf{x}_{t}+\mathbf{A}_{2}^{(1)} \mathbf{x}_{t-1}+\mathbf{A}_{3}^{(1)} \mathbf{x}_{t-2}, & \text { for } i_{t+1}=1 \\ \boldsymbol{\mu}^{(2)}+\mathbf{A}_{1}^{(2)} \mathbf{x}_{t}+\mathbf{A}_{2}^{(2)} \mathbf{x}_{t-1}+\mathbf{A}_{3}^{(2)} \mathbf{x}_{t-2}, & \text { for } i_{t+1}=2 \\ \boldsymbol{\mu}^{(3)}+\mathbf{A}_{1}^{(3)} \mathbf{x}_{t}+\mathbf{A}_{2}^{(3)} \mathbf{x}_{t-1}+\mathbf{A}_{3}^{(3)} \mathbf{x}_{t-2}, & \text { for } i_{t+1}=3\end{cases}
$$

where $i_{t}$ is the fitted state assignment given by Eq. (7). The cluster means $\boldsymbol{\mu}^{(1)}, \boldsymbol{\mu}^{(2)}, \boldsymbol{\mu}^{(3)}$ and parameter matrices $\mathbf{A}_{i}^{(k)}$ for $i, k \in\{1,2,3\}$ are constant. Note that, by constructing the model in such a way, the dynamics will change in the time step prior to a transition in the affiliation sequence.

We are interested in whether the dynamical properties of the resulting model from the FEMBV-VAR framework can show any insight on the mechanisms characterizing transitions between states and whether the reduced dynamical model exhibits properties that are physically plausible. In particular, we would like to see if the increased finite-time instability during blocking events (Schubert and Lucarini 2015, 2016; Faranda et al. 2017; Lucarini and Gritsun 2020) and loss of hyperbolicity in transitions between zonal and blocked states (Lucarini and Gritsun 2020) manifest at all in the FEM-BV-VAR reduced model defined by Eq. (9).

In order to study the dynamics we use the resulting affiliation sequences and parameter matrices from the optimal FEM-BV-VAR model to construct the following system:

$$
\left[\begin{array}{c}
\mathbf{x}_{t+1} \\
\mathbf{x}_{t} \\
\mathbf{x}_{t-1}
\end{array}\right]=\left[\begin{array}{ccc}
\mathbf{A}_{1}^{\left(i_{t+1}\right)} & \mathbf{A}_{2}^{\left(i_{t+1}\right)} & \mathbf{A}_{3}^{\left(i_{t+1}\right)} \\
\mathbf{I} & \mathbf{0} & \mathbf{0} \\
\mathbf{0} & \mathbf{I} & \mathbf{0}
\end{array}\right]\left[\begin{array}{c}
\mathbf{x}_{t} \\
\mathbf{x}_{t-1} \\
\mathbf{x}_{t-2}
\end{array}\right] .
$$

Eq. (10) describes a discrete linear mapping system governing the tangent dynamics of Eq. (9), with a finite number of transitions between states defined a priori by the switching sequence Eq. (7). As we retain the leading $d=20 \mathrm{PCs}$, the system Eq. (10) has a 60-dimensional state space. The matrices I are 20-dimensional identity matrices, and $\mathbf{0}$ denotes the $20 \times 20$ zero matrix. Through 
Eq. (10) we can define the linear propagator $\mathbf{A}(t)$ of the tangent dynamics:

$$
\mathbf{A}(t):=\left[\begin{array}{ccc}
\mathbf{A}_{1}^{\left(i_{t+1}\right)} & \mathbf{A}_{2}^{\left(i_{t+1}\right)} & \mathbf{A}_{3}^{\left(i_{t+1}\right)} \\
\mathbf{I} & \mathbf{0} & \mathbf{0} \\
\mathbf{0} & \mathbf{I} & \mathbf{0}
\end{array}\right] .
$$

The linear propagator can be used to construct the matrix cocycle $\mathcal{A}(t, \tau)$, that is, the forward and backward mapping of solutions under the tangent dynamics. The variable $\tau$ represents the window over which the cocycle is defined starting from time $t$. In other words, $\mathcal{A}(t, \tau)$ is defined as compositions of the linear propagator in time:

$$
\mathcal{A}(t, \tau)=\mathbf{A}(t+\tau) \ldots \mathbf{A}(t+1) \mathbf{A}(t) .
$$

Eq. (12) expresses the cocycle for $\tau>1$, however the construction is similar for $\tau \leq 1$.

The matrix cocycle is an integral part of the Multiplicative Ergodic Theorem (Oseledets 1968) which defines the asymptotic growth and decay rates, or Lyapunov exponents, of a dynamical system. The theorem states that, under suitable assumptions, for a cocycle operating on a phase space of dimension $N$, there exists a unique set of subspaces $\left\{\Phi_{i}(t)\right\}(i \in 1, \ldots, m$ where $m \leq N)$ which are covariant under the tangent dynamics, and all vectors $\mathbf{v}$ which lie in the subspace have the same asymptotic growth or decay rate. The Lyapunov exponent $\lambda_{i}$ of subspace $\Phi_{i}$ is then defined by

$$
\lambda_{i}=\lim _{\tau \rightarrow \infty} \frac{1}{\tau} \log \|\mathcal{A}(t, \tau) \mathbf{v}\| \quad \text { iff } \quad \mathbf{v} \in \Phi_{i}(t) \backslash \Phi_{i+1}(t) .
$$

Each subspace $\Phi_{i}$ is spanned by a set of vectors $\left\{\phi_{i}(t)\right\}$ called covariant Lyapunov vectors which grow with rate $\lambda_{i}$ forward and $-\lambda_{i}$ backward in time under the tangent linear propagator (Pazó et al. 2008). Unlike forward and backward Lyapunov vectors, the CLVs are norm-independent, give the local directions of growth and decay in tangent space, and generally are non-orthogonal. 
While forward and backward Lyapunov vectors characterize the global geometry, CLVs are useful for understanding the local geometry of the tangent space in a dynamical system.

We calculate the CLVs using algorithm 2.2 from Froyland et al. (2013), which is also summarized in Quinn et al. (2020). The calculation is based on the proof of the extension of the Multiplicative Ergodic Theorem to non-invertible linear propagators (Froyland et al. 2010, Theorem 4.1). The $i$-th eigenspace of $\mathcal{A}(t-M, M)^{*} \mathcal{A}(t-M, M)$ (where the star denotes the adjoint) pushed forward by the matrix cocycle $\mathcal{A}(t-M, M)$ is equal to $\Phi_{i}(t)$ when $M \rightarrow \infty$. The right singular vectors of $\mathcal{A}(t-M, M)$ are equivalent to the eigenvectors of $\mathcal{A}(t-M, M)^{*} \mathcal{A}(t-M, M)$. The general idea then to compute the CLV at time $t$ is that one calculates the $i$-th right singular vector $\eta_{i}$ for the cocycle $\mathcal{A}(t-M, M)$ and then pushes forward $\eta_{i}$ by $M$ time steps using the tangent linear propagator. We therefore refer to $M$ as the push forward step. In order to prevent the collapse of sub-leading vectors onto the leading vector, for each $i>1$ we take an orthogonal projection onto the right singular vectors $\eta_{j}$ of $\mathcal{A}(t-M+n k, M)$ where $j=1, \ldots, i-1$. Here $k=1, \ldots, \frac{M}{n}$ and $n$ is the time step for the orthogonal projection. Due to the rapid switching between states observed at times, we use $n=1$ day. This will approximate $\phi_{i}(t)$ only if the push forward step $M$ is sufficiently large. If $M$ is small, we refer to the resulting vector as a "mixed singular vector" (MSV). The condition "sufficiently large" is not known a priori for the system, so we analyse the following range of push forward steps: $M=3,10,30,50$ days.

In the following sections we investigate the growth rates and alignment of the leading CLVs, and discuss how we use these to differentiate between MSVs and CLVs. We compare the behavior for the different push forward steps and analyze how changes in either property relates to transitions between the states. 


\section{a. Finite-time exponents}

The first property of the vectors that we analyze is their finite-time growth rates, i.e., finite-time exponents (FTEs). Due to the rapid transitioning between states, we consider the growth rates over the course of one day. We define the FTEs as in Wolfe and Samelson (2007), here Eq. (14a). To calculate the FTEs we use a forward difference approximation to the derivative, which in our case simplifies to applying the linear propagator to the vector calculated for a given day and taking the difference of the $\mathrm{L}^{2}$-norms:

$$
\begin{aligned}
\Lambda_{i}(t) & =\frac{1}{\left\|\phi_{i}(t)\right\|} \frac{d}{d t}\left\|\phi_{i}(t)\right\| \\
& =\left\|\mathcal{A}(t, 0) \phi_{i}(t)\right\|-\left\|\phi_{i}(t)\right\| .
\end{aligned}
$$

Note that $\left\|\phi_{i}(t)\right\|=1$ for the vectors computed using the Froyland et al. (2013) algorithm and therefore the scaling factor is omitted from Eq. (14b).

In order to differentiate between MSVs and CLVs, we compare the FTEs computed using Eq. (14b) to the approximate asymptotic Lyapunov exponents computed from the QR decomposition method (appendix C). If the vector is a CLV then the averages of the FTEs over many time intervals should converge to the asymptotic Lyapunov exponents (Kuptsov and Parlitz 2012). For the computation of the asymptotic growth rates we use the full matrix cocycle over the period of the FEM-BV-VAR fit and an orthonormalization time step of 1 day. We find that asymptotically the model is stable and there is little evidence of a spectral gap in the leading exponents. FIG. 4 plots the asymptotic exponents compared to the statistics of the FTEs calculated for each push forward step. For $M=3$ the averages of the leading FTEs do not match well with the approximate asymptotic values. We therefore label the vectors computed for $M=3$ as MSVs. It can be seen that as the push forward step is increased, the mean FTEs approach the asymptotic values and the standard deviation decreases for the leading growth rates. Since the finite-time and asymptotic growth rates 
are computed using different methods, this agreement provides confidence in the accuracy of the CLV calculation for $M=10,30$, and 50 .

To quantify the total transient growth at each time step in an asymptotically stable system, we use a finite-time variant of the Kaplan-Yorke dimension as a measure introduced in Quinn et al. (2020). As a first step we reorder the FTEs as

$$
\max \left(\Lambda_{i}(t)\right)>\cdots>\min \left(\Lambda_{i}(t)\right)=\tilde{\Lambda}_{1}(t)>\cdots>\tilde{\Lambda}_{N}(t)
$$

The finite-time dimension measure can be computed as

$$
\operatorname{dim}_{K Y}(t)=j+\frac{\sum_{i=1}^{j} \tilde{\Lambda}_{i}(t)}{\left|\tilde{\Lambda}_{j+1}(t)\right|},
$$

where $j \in\{1, \ldots, N\}$ is the largest index which satisfies the conditions

$$
\sum_{i=1}^{j} \tilde{\Lambda}_{i}(t) \geq 0 \text { and } \sum_{i=1}^{j+1} \tilde{\Lambda}_{i}(t)<0 .
$$

It is important to note that the sums of the FTEs do not relate to typical expansion and contraction of volumes in tangent space as the MSVs and CLVs are not necessarily orthogonal (Kuptsov and Kuznetsov 2018). The individual FTEs give the specific expansion and contraction of the tangent vectors, and the finite-time dimension measure Eq. (16) defined as the local Kaplan-Yorke dimension is being used here as an approximate measure of the number of unstable and near-neutral FTEs.

We next compare the probability of the occurrence of a positive dimension across all push forward steps. The short push forward of $M=3$ shows the most unstable behavior, with $73 \%$ of time instances associated with positive FTEs. The largest probability of occurrence is in the negative NAO state with $99 \%$ of days assigned to that state experiencing a positive FTE. This is followed by the positive NAO state at $62 \%$ and then the Atlantic Ridge at $39 \%$. The probabilities of observing a positive FTE starkly drops for the longer push forwards $M=10,30,50$ with all at 
less than $1 \%$ regardless of state. This suggests that the instabilities within this model are associated with fast-scale dynamics that are filtered out when using longer push forward lengths. On short time scales the model is unstable the majority of the time, while on long time scales the stable dynamics of the model dominate.

For the $M=3$ case exhibiting the most unstable behavior, we are interested in characterizing stability based on the finite-time dimension, $\overline{\operatorname{dim}_{K Y}(t)}$, where the overbar denotes a conditional average over residency in each state (shown in TABLE 4). We see that the $\mathrm{NAO}^{-}$state shows the most unstable behavior, followed by the $\mathrm{NAO}^{+}$and then the $\mathrm{AR}$ state. To filter out periods of rapid transitioning, we also consider the average dimension of persistent states. Here we use a 5-day filter in which we include in the average only days where the model was in the state both 2 days before and 2 days following the day on which the dimension was calculated. When only persistent events are considered, the AR state experiences no unstable behavior, while the average dimension has increased slightly for both NAO phases. This is in agreement with previous studies that show blocking events (typically associated with a negative NAO phase) tend to have higher instantaneous instability than times of strong zonal flow (typically associated with the positive NAO phase) (Schubert and Lucarini 2016; Faranda et al. 2016, 2017; Lucarini and Gritsun 2020). Since the FTEs correspond to the growth and decay rates of particular MSVs, we can identify the modes which experience finite-time growth in each persistent state. Given that the average $\operatorname{dim}_{K Y}(t)$ measure is 0 in the AR state we can conclude there is no growing mode during long residencies in that state. For both the $\mathrm{NAO}^{-}$and the $\mathrm{NAO}^{+}$state there is only one unstable mode that contributes to the positive $\operatorname{dim}_{K Y}(t)$ measure. To visualize what these modes look like in physical space, we take a projection of the MSVs onto the corresponding EOFs (appendix A). The resulting patterns are shown in FIG. 5. For the $\mathrm{NAO}^{-}$state the instability arises in MSV 1 and projects as the NAO pattern itself, with a larger magnitude anomaly to the southeast of Greenland and an 
opposite, smaller magnitude anomaly south of that stretching from the east coast of North America to Spain. We see a similar pattern emerging in MSV 2 for the $\mathrm{NAO}^{+}$state, with the northern anomaly stretching west into the northern parts of Canada and having a smaller magnitude.

We are also interested in the unstable MSVs around transitions and whether or not the patterns are distinct from those in FIG. 5. We first identify all transitions associated with persistent states, i.e., residencies of greater than 4 days both before and after the transition. For this residency length and a push forward of $M=3$ days, each of the 6 distinct transitions will have the same progression of dynamics each time the model experiences that particular transition. We show these 6 progressions of MSV patterns, FTEs, and alignment (introduced in the next section) in appendix D. While these transitions between persistent states account for some 921 days with unstable exponents over the full fit period, we find that this corresponds to only a few dozen distinct, recurring unstable patterns. By further classifying the observed patterns using the pattern correlation between MSVs, we determine four distinct modes that experience finite-time growth around the time of a transition (shown in FIG. 6). The main feature of all of these unstable modes compared to the unstable modes within the persistent states is more zonally oriented anomalous pressure gradients. TABLE 5 lists the transitions in which each pattern occurs, the day on which it occurs, the MSV number and associated FTE value. Patterns A and B appear only in transitions from the $\mathrm{NAO}^{-}$state, pattern C only appears in transitions from the $\mathrm{NAO}^{+}$to the $\mathrm{AR}$ state, and pattern $\mathrm{D}$ appears in both $\mathrm{NAO}^{-}$ to $\mathrm{NAO}^{+}$and $\mathrm{NAO}^{+}$to $\mathrm{NAO}^{-}$transitions. In terms of the MSVs in which the unstable patterns are expressed, patterns B and C are solely associated with MSV 2, pattern D is solely associated with MSV 1, and pattern A occurs in both MSV 1 and 2. All unstable patterns occur either on the first or second day the model is in the end state of the transition. We note here that none of these patterns occur in transitions from the AR state. In those two cases the transition is marked by the emergence of the unstable persistent patterns in FIG. 5 in either MSV 1 or 2 as dictated by the 
end state. The MSV patterns associated with transitions to and from the respective NAO states are associated with either the formation or decay of the meridionally oriented structures characteristic of the respective NAO phases.

\section{b. Alignment of MSVs and CLVs}

While the FTEs give the relative growth and decay rates of tangent vectors to the subspaces, the angle between the vectors (otherwise known as alignment) gives an idea of transversality of the subspaces (Kuptsov and Kuznetsov 2018). High alignment of CLVs, or a vanishing angle between subspaces, has been suggested to be an indicator of transitions and catastrophic events (Beims and Gallas 2016; Sharafi et al. 2017). This would also agree with the loss of hyperbolicity when transitioning between unstable periodic orbits with differing numbers of unstable dimensions, as was found to be the case for zonal vs blocked states in Lucarini and Gritsun (2020). We measure the alignment of two vectors through $\theta_{i, j}=\left|\cos \left(\Theta_{i, j}\right)\right|$ where $\Theta_{i, j}$ is the angle between the $i$-th and $j$-th vector. Values of $\theta_{i, j}$ close to one imply high alignment of the MSVs or CLVs, while values close to zero imply orthogonality. Here we calculate the alignment using the following:

$$
\theta_{i, j}(t)=\frac{\left|\phi_{i}(t) \cdot \phi_{j}(t)\right|}{\left\|\phi_{i}(t)\right\| \cdot\left\|\phi_{j}(t)\right\|}
$$

We first consider the alignment of the MSVs calculated for the short push forward step $(M=3)$. FIG. 7 shows the alignment of the leading MSVs $\left(\theta_{1,2}, \theta_{2,3}\right.$, and $\left.\theta_{1,3}\right)$ for two different time segments; we also plot the leading growth rates $\left(\Lambda_{1}, \Lambda_{2}\right.$, and $\left.\Lambda_{3}\right)$, dimension, and state indicators for comparison. We indeed see a spike in the alignment values around the time of transitions, with the most prominent spikes typically in $\theta_{1,2}$ and $\theta_{2,3}$. The differing behavior of dimension by state discussed in section 3a can be seen clearly in the two figures. FIG. 7a shows an example segment which has long residencies in the $\mathrm{NAO}^{-}$state. We see that for long enough residencies 
the dimension measure remains around 3 with the driving instability coming from the first MSV. On the contrary, residencies longer than two days in the AR state show the dimension measure quickly dropping to zero. This is further illustrated in FIG. $7 \mathrm{~b}$ where the model resides primarily in the $\mathrm{AR}$ and $\mathrm{NAO}^{+}$state. The lower dimension measures are driven by the differing behavior of $\Lambda_{1}$ which remains close to $\Lambda_{2}$ and both oscillate around zero. We see that for long enough residency in the $\mathrm{NAO}^{+}$state the instability is driven by $\Lambda_{2}$ overtaking $\Lambda_{1}$.

In order to obtain a more complete understanding of the alignment behavior around transitions, FIG. 8 shows the collective alignment values centered around the days associated with transition (filtered for state residencies longer than 4 days before and after the transition). The transition occurs from day 0 to day 1 . The greatest change in behavior can be seen on days 0,1 , and 2 for $\theta_{1,2}$, and days 1 and 2 for $\theta_{2,3}$ and $\theta_{1,3}$. The most noticeable change is in the increased values of the third quartile and the maximum. The leading alignment $\theta_{1,2}$ shows an overall increase in alignment values on day 1 and 2 for all transitions. There is also an increase in the median value preceding the transitions on day -1 . The increased spread of alignment around transitions is due to differing alignment behavior for each type of transition as can be seen in FIG. 7. We therefore separate the alignment behavior by specific transition and plot the ensemble of trajectories in FIG. 9. We see that transitions from the $\mathrm{NAO}^{-}$state show an increase in $\theta_{1,2}$ on the days preceding the transition. The peak in $\theta_{1,2}$ occurs on the last day the affiliation sequence is in the preceding state. We also observe that there is a spike in $\theta_{2,3}$ following both transitions from the $\mathrm{NAO}^{-}$state; for $\mathrm{NAO}^{-}$ to $\mathrm{AR}$ it occurs on the day following the peak in $\theta_{1,2}$ and for $\mathrm{NAO}^{-}$to $\mathrm{NAO}^{+}$it occurs two days following. For both transitions from the $\mathrm{NAO}^{+}$state there is an increase in $\theta_{1,2}, \theta_{2,3}$, and $\theta_{1,3}$, with the maximum values for each occurring two days after the transitions. For the AR to $\mathrm{NAO}^{+}$ transition there is an increase in $\theta_{2,3}$ with a peak on the day just following the transition. The other two alignments $\left(\theta_{1,2}\right.$ and $\left.\theta_{1,3}\right)$ also show a weak increase. The AR to $\mathrm{NAO}^{-}$transition shows the 
overall weakest signal in alignment, although all three still display an increase within two days of the transition.

Next we consider the behavior of the alignment of the leading two MSVs or CLVs, $\theta_{1,2}(t)$, across the varying push forward lengths. This is displayed in the panels of Fig. 10a. The first difference we notice is in the timescale of variability of the alignment. For shorter push forward lengths we observe that large changes in alignment occur more often than for longer push forward lengths. We also observe the emergence of a low-frequency signal within the variability as the push forward length is increased. To explore the emergence of this signal we compute the power spectral density (PSD) of each alignment time series. The PSDs are shown in FIg. 10b, scaled to show the frequency percentage contribution to variance. The red dots show the peaks that are identified using a threshold of 2 standard deviations away from neighboring measures, while the red crosses use a threshold of 3 standard deviations. We can see the emergence of a significant low-frequency signal for the push forward length of 30 days or longer. This frequency corresponds to a period of approximately 1 year.

We relate the annual signal emerging in the alignment of the leading CLVs to the seasonality of the NAO. A study of the NAO in both observational data and reanalysis products has shown that there is increased variability in the NAO index in the boreal winter and decreased average NAO values in the boreal summer (Hanna et al. 2015). To measure relative variability in the NAO index for our model we define a transition index,

$$
\text { Transition index }=\sum_{i=t-50}^{t} \frac{\mathbb{I}_{\text {tran }}(i)}{50}
$$

Here $\mathbb{I}_{\text {tran }}(i)$ is again the indicator function for a transition occurring at time $i$, and we choose a window of 50 days to match the longest push forward step used to calculate alignment. The time series of the transition index compared to $\theta_{1,2}$ for $M=50$ is shown in FIG. 11 . We observe that the 
two measures are anti-correlated. The maximum Pearson correlation coefficient is -0.45 at a 17 day lag with the alignment. The transition index also shows a peak in its PSD corresponding to an annual signal (not shown).

While FIG. 11 compares the alignment and NAO variability in time, we are also interested in the average behavior by season. The various NAO indices computed from both observational records and reanalysis products have been shown to exhibit distinct seasonal behavior. In a study by Hanna et al. (2015) the authors analyze a collection of station-based data and reanalyses and compare seasonal differences as well as trends. They find that there has been increased variability in the NAO during the boreal winter (DJF), particularly in December, throughout the last century. The authors also noted a decrease in boreal summer (JJA) NAO values over the past 20-30 years. To analyze how the seasonality of our model compares, we consider the total number of transitions and days spent in a given state each season as shown in TABLE 2 . The seasonality in the $\mathrm{NAO}^{-}$state is seen more through the total number of days spent in a given state and average residency times. As mentioned in section $2 \mathrm{c}$, the $\mathrm{NAO}^{-}$state accounts for $46.5 \%$ of the total number of model days. The largest contribution to that comes from JJA (41\%) compared to DJF which only accounts for $11 \%$ of $\mathrm{NAO}^{-}$days. This seasonality is similar to, but much more pronounced than, that observed for the CPC NAO index; over the same period as the model fit, $45 \%$ of days had a negative daily mean index, and $20 \%$ of these days occurred during DJF compared to $29 \%$ accounted for by JJA. The average residency length also has a seasonal signal (TABLE 1), with its maximum in JJA (9.3 days) and minimum in DJF (2.5 days). We observe as expected a seasonal signal in the transition probabilities, with the highest probability of a transition occurring in SON (30\%), while JJA has the lowest overall probability of transitions (15\%). When we separate by the state associated with each transition, we see different seasonal behavior across the three states. Transitions associated with the $\mathrm{NAO}^{-}$state have roughly the same probability of occurring in DJF as in the JJA (16\%). 
Those probabilities are lower than what is seen in MAM $(23 \%)$ and SON $(24 \%)$ which are generally referred to as transitional seasons. On the contrary, the transitions associated solely with the $\mathrm{NAO}^{+}$ and Atlantic Ridge states have a much stronger seasonal signal. The probability is nine times higher in DJF (18\%) than in JJA (2\%) for transitions between the $\mathrm{NAO}^{+}$and $\mathrm{AR}$ states which contributes to the overall increase in DJF variability compared to JJA.

We now turn to the average behavior of alignment by season. FIG. 12 shows the alignment averaged over each season of the indicated pairs of CLVs. We see a clear seasonal behavior of $\theta_{1,2}$ with a maximum in summer and a minimum in autumn and winter. Interestingly, there is also a seasonal signal in $\theta_{2,3}, \theta_{2,4}$ and $\theta_{3,4}$ (although weaker for $\theta_{2,4}$ and $\theta_{3,4}$ ). We do not see a seasonal cycle in the alignments with the more asymptotically stable CLVs (5-7) as their dominant signals have a cycle length of less than a year.

\section{Summary}

We have presented here a dynamical analysis of a reduced model for the NAO teleconnection. The preferred model has been constructed through application of the FEM-BV-VAR method which has been previously used to identify atmospheric pressure states consistent with known coherent features in the North Atlantic (Risbey et al. 2015; O'Kane et al. 2017). The identified states are also consistent with an alternate FEM-BV-EOF (Franzke et al. 2009) variant analysis. Using the NCEP/NCAR Reanalysis 1 (Kalnay et al. 1996) from 1979 to 2018, we tested a range of hyperparameters to determine an optimal model. The resulting optimal model was found to be non-Markovian with a time dependence (memory) of 3 days, an average state length of 5 days, and 3 cluster states. The cluster states closely resemble the two phases of the NAO and a pattern similar to the AR. 
In order to study the time-dependent model dynamics, we constructed a discrete linear mapping system defined on a delay-embedding of the PCs. The switching is defined a priori by the affiliation sequence resulting from the FEM-BV-VAR fit. Through this novel way of constructing the system we were able to analyze the time-dependent tangent linear propagator, calculating MSVs and CLVs, their finite-time growth and decay rates, and their alignment. We differentiate between short time-scale dynamics and long time-scale dynamics by using different window lengths over which to calculate the vectors.

While the individual states are asymptotically stable, on short time-scales they can exhibit finitetime growth. In particular, we found that both NAO states contain finite-time unstable MSVs for a window length of 3 days, with the $\mathrm{NAO}^{-}$state showing stronger instability than the $\mathrm{NAO}^{+}$ state. We used a finite-time dimension measure to characterize the instability and identified the largest dimension to be associated with the blocked $\mathrm{NAO}^{-}$state, which is consistent with recent studies of blocking in theoretical models (Schubert and Lucarini 2016) and data (Faranda et al. 2017; Lucarini and Gritsun 2020). These findings provide a new interpretation regarding the predictability of blocking events. While the blocked state is conventionally thought of as having higher predictability for weather conditions, the increased instability associated with such states as found in Schubert and Lucarini (2016); Faranda et al. (2017); Lucarini and Gritsun (2020) and the study at hand provide a new insight as to why models struggle to capture the onset and decay of blocking events. We also projected the unstable MSVs into physical space in order to visualize the pressure anomaly patterns associated with the finite-time growth. During persistent states the instability manifests as an NAO-like meridional pressure gradient, whereas around transitions between persistent states the instability manifests in more zonally oriented pressure gradient patterns. 
The alignment of the vectors also showed different behavior on short versus long time-scales.

On short time-scales (window length of 3 days) there was an increase in alignment of the leading MSVs around the time of transitions. The increase occurred anywhere between the last day of the preceding state and the second day of the end state. Such an increase in alignment can be related to the loss of hyperbolicity observed in transitions between unstable periodic orbits, supporting the results of Lucarini and Gritsun (2020) that identified unstable periodic orbits associated with blocking as having on average a higher dimension than those associated with strong zonal flow. For the longer time-scale CLVs we observed starkly different behavior whereby a low-frequency signal in alignment emerged as the window length was increased, converging to an annual oscillation with a maximum in the boreal summer (JJA) and a minimum in the boreal winter (DJF) at windows of 30 plus days. A transition index, defined over the same window length, was computed to characterize the tendency of the model to switch between states and found to be anti-correlated with the alignment and have a pronounced annual signal. The seasonality in alignment was also related to the seasonality seen in the $\mathrm{NAO}^{-}$average residency length and model preference for different states in JJA versus DJF.

The novel dynamical systems analysis of a data-driven model of the NAO presented here is general and does not have to be restricted to this particular phenomenon nor to atmospheric teleconnection studies. One could perform a similar analysis on any resulting model from the use of the FEM-BV-VAR clustering method or general reduced order stochastic models. With respect to atmospheric and oceanic teleconnections, this method provides a way of extracting the large-scale unstable perturbation directions associated with specific phenomena. Future studies will aim to characterize the behavior of other teleconnection interactions as well as anomalous events associated with particular large-scale atmospheric modes. 
Acknowledgments. CQ and TO are supported by the Australian Commonwealth Scientific and Industrial Research Organisation (CSIRO) Decadal Climate Forecasting Project (https: //research.csiro.au/dfp). DH is supported by the CSIRO through a ResearchPlus postdoctoral fellowship. The EOF analysis was implemented using the scikit-learn Python package (Pedregosa et al. 2011), and plots were generated using the Python package Matplotlib (Hunter 2007).

Data availability statement. The NCEP/NCAR reanalysis output used is provided by the NOAA/OAR/ESRL PSL, Boulder, Colorado, USA, and may be accessed at https://psl noaa. gov/data/reanalysis/reanalysis.shtml. All source code used to perform the analyses presented in this study may be found at https://doi.org/10.5281/zenodo. 4035644 .

\section{APPENDIX A}

\section{EOFs of North Atlantic Region}

FIG. A1 shows the EOFs used in the dimension reduction applied to the NCEP/NCAR Reanalysis 1 atmospheric pressure anomaly data from the base period 1 January 1979 to 31 December 2018. In calculating the EOFs and corresponding PCs, the data is weighted by the square root of the cosine of the latitude. We use a truncated singular value decomposition for 200 components and a unit normalization for the EOFs. The 20 EOFs displayed in Fig. A1 account for $91 \%$ of the total variance, and EOF 1 resembles the typical NAO pattern.

APPENDIX B 
In general, direct minimization of Eq. (2) with the component losses given by Eq. (6) to find the optimal affiliations $\boldsymbol{\Gamma}$ and parameters $\boldsymbol{\Theta}$ is not practical. However, the loss function is separately convex in $\boldsymbol{\Gamma}$ and $\boldsymbol{\Theta}$, and approximate minimizers $(\hat{\boldsymbol{\Gamma}}, \hat{\boldsymbol{\Theta}})$ may be straightforwardly computed by alternately minimizing Eq. (2) with respect to $\boldsymbol{\Gamma}$ for fixed $\boldsymbol{\Theta}$ and vice versa, until convergence is reached. The minimization problem with respect to $\boldsymbol{\Gamma}$ for fixed $\boldsymbol{\Theta}$ may be formulated as a constrained linear programming problem (Metzner et al. 2012) and solved numerically. For fixed $\boldsymbol{\Gamma}$, the optimal parameters $\Theta_{i}$ are given by weighted least-squares estimates. In terms of the matrices

$$
\begin{aligned}
\mathbf{X} & =\left(\mathbf{x}_{m_{\max }+1}, \ldots, \mathbf{x}_{T}\right) \in \mathbb{R}^{d \times\left(T-m_{\max }\right)}, \\
\mathbf{Z} & =\left(\begin{array}{ccc}
1 & \ldots & 1 \\
\mathbf{x}_{m_{\max }} & \ldots & \mathbf{x}_{T-1} \\
\vdots & \ldots & \vdots \\
\mathbf{x}_{m_{\max }-m} & \ldots & \mathbf{x}_{T-m}
\end{array}\right) \in \mathbb{R}^{(1+m d) \times\left(T-m_{\max }\right)}, \\
\mathbf{W}_{i} & =\operatorname{diag}\left(\left[\gamma_{m_{\max }+1}\right]_{i}, \ldots,\left[\gamma_{T}\right]_{i}\right) \in \mathbb{R}^{\left(T-m_{\max }\right) \times\left(T-m_{\max }\right)}, \\
\mathbf{B}_{i} & =\left(\boldsymbol{\mu}^{(i)}, \mathbf{A}_{1}^{(i)}, \ldots, \mathbf{A}_{m}^{(i)}\right) \in \mathbb{R}^{d \times(1+m d)},
\end{aligned}
$$

the estimated parameters for state $i$ at fixed $\boldsymbol{\Gamma}$ may be compactly written as

$$
\begin{aligned}
\hat{\mathbf{B}}_{i} & =\mathbf{X} \mathbf{W}_{i} \mathbf{Z}^{T}\left(\mathbf{z} \mathbf{W}_{i} \mathbf{Z}^{T}\right)^{-1}, \\
\hat{\mathbf{\Sigma}}^{(i)} & =\frac{1}{\operatorname{Tr}\left[\mathbf{W}_{i}\right]}\left(\mathbf{X}-\hat{\mathbf{B}}_{i} \mathbf{Z}\right) \mathbf{W}_{i}\left(\mathbf{X}-\hat{\mathbf{B}}_{i} \mathbf{Z}\right)^{T},
\end{aligned}
$$

where $\operatorname{Tr}[\mathbf{A}]$ denotes the trace of a matrix $\mathbf{A}$. This coordinate descent method finds a local minimum of the loss function for a given initial guess at the optimal parameters and not necessarily a globally optimal solution. In order to reduce the degree to which this occurs, in all of the results presented we run the optimization $N_{\text {init }}=20$ times with different initial guesses and keep the solution with the lowest loss. 
To select a single set of values for the hyperparameters $K, m$, and $p$, we use the following cross-validation method. The observed sample is divided into $N_{\text {fold }}+1$ approximately equal length segments $\mathcal{T}_{1}, \ldots, \mathcal{T}_{N_{\text {fold }}+1}$, and each model is refit $N_{\text {fold }}$ times, where on the $i^{\text {th }}$ iteration the first $i$ segments are used as the training sample. Holding the obtained state parameters $\hat{\boldsymbol{\Theta}}$ fixed, the optimal affiliations are calculated by minimizing the cost function evaluated over the $(i+1)^{\text {th }}$ segment, adjusting the upper bound $C_{T}$ as appropriate for the length of the segment with fixed $p$. The weighted root mean square error

$$
\operatorname{RMSE}_{i}=\sqrt{\frac{1}{d\left(T_{i}-m_{\max }\right)} \sum_{t \in \mathcal{T}_{i+1}} \sum_{j=1}^{K}\left[\boldsymbol{\gamma}_{t}\right]_{j}\left\|\mathbf{x}_{t}-\hat{\mathbf{x}}_{t}^{(j)}\right\|^{2}}
$$

is then evaluated for each test segment, where $\hat{\mathbf{x}}_{t}^{(j)}$ denotes the expected value under state $j$. The mean reconstruction RMSE over the set of test sets provides a measure of the model's ability to generalize to future data, which we use in lieu of estimates of out-of-sample prediction error, with good performance on this measure involving a compromise between model flexibility and overfitting the training data. We note that the more standard cross-validation approach, that is estimation of the out-of-sample forecast error, would require an additional model for the dynamics of the hidden switching process, which we here leave to future work. Alternatively, in-sample measures based on information criteria could be used when combined with an appropriate likelihood model. However, this similarly requires an appropriate probabilistic model to be specified for the switching and noise processes, and, moreover, the very large number of estimated degrees of freedom in comparison to the available sample size may lead to concerns as to their suitability (Burnham and Anderson 2002). 
The QR algorithm we use for computing the leading asymptotic Lyapunov exponents follows Dieci et al. (1997). It is based on the numerical linear algebra factorisation of a matrix into an orthogonal matrix $\mathbf{Q}$ and an upper triangular matrix $\mathbf{R}$. The initial arbitrary orthogonal matrix can be set as $\mathbf{Q}_{0}=\mathbf{I}_{N}$ where $\mathbf{I}$ is the identity matrix and $N$ is the number of states in the state space. We then define the $\mathbf{Q}_{i}$ and $\mathbf{R}_{i}$ matrices iteratively through the $\mathrm{QR}$ decomposition of $\mathbf{A}_{i} \mathbf{Q}_{i-1}$ :

$$
\mathbf{Q}_{i} \mathbf{R}_{i}=\mathbf{A}_{i} \mathbf{Q}_{i-1},
$$

where $\mathbf{A}_{i}=\mathbf{A}\left(t_{i}\right)$, our tangent linear propagator defined by Eq. (11). The upper triangular matrix $\mathbf{R}_{i}$ holds the eigenvalues $R_{i, j j}>0$ where $j j$ indicates the position of the matrix entry. After $T$ time steps we have the equivalence

$$
\mathbf{Q}_{T} \mathbf{R}_{T} \ldots \mathbf{R}_{1}=\mathbf{A}_{T} \ldots \mathbf{A}_{1} \mathbf{Q}_{0}
$$

We then approximate the asymptotic Lyapunov exponents through

$$
\lambda_{j}=\frac{1}{T} \sum_{i=1}^{T} \ln R_{i, j j} \quad \text { for } j=1, \ldots, N .
$$

\section{APPENDIX D}

\section{CLV patterns for transitions associated with persistent states}

We show the leading CLV patterns during each of the six transitions associated with persistent states: $\mathrm{AR}$ to $\mathrm{NAO}^{-}$(FIG. D1), $\mathrm{AR}$ to $\mathrm{NAO}^{+}$(FIG. D2), $\mathrm{NAO}^{-}$to $\mathrm{AR}$ (FIG. D3), $\mathrm{NAO}^{-}$to $\mathrm{NAO}^{+}$ (FIg. D4), $\mathrm{NAO}^{+}$to AR (FIg. D5), $\mathrm{NAO}^{+}$to $\mathrm{NAO}^{-}$(FIG. D6). The transition occurs between Day 0 and 1 , and we show the three days preceding and the 3 days following. Due to the filtering on persistent states (minimum of 5 days in each state on either side of the transition), Days -2 and 3 show the CLV patterns associated with the stationary states before and after the transition, respectively. The top two panels in each figure indicate the associated alignment and FTE behavior. Note that we only show $\Lambda_{1}$ and $\Lambda_{2}$ as $\Lambda_{3}$ is always negative in these cases. 


\section{References}

Barnston, A. G., and R. E. Livezey, 1987: Classification, Seasonality and Persistence of LowFrequency Atmospheric Circulation Patterns. Monthly Weather Review, 115 (6), 1083-1126, doi:10.1175/1520-0493(1987)115<1083:CSAPOL>2.0.CO;2.

Beims, M. W., and J. A. Gallas, 2016: Alignment of lyapunov vectors: A quantitative criterion to predict catastrophes? Scientific reports, 6 (1), 1-7, doi:10.1038/srep37102.

Benedict, J. J., S. Lee, and S. B. Feldstein, 2004: Synoptic view of the north atlantic oscillation. Journal of the Atmospheric Sciences, 61 (2), 121-144, doi:10.1175/1520-0469(2004)061\% 3C0121:SVOTNA\%3E2.0.CO;2.

Burnham, K. P., and D. R. Anderson, 2002: Model Selection and Multimodel Inference. 2nd ed., Springer.

Cassou, C., 2008: Intraseasonal interaction between the madden-julian oscillation and the north atlantic oscillation. Nature, 455 (7212), 523-527, doi:10.1038/nature07286.

Cassou, C., L. Terray, and A. S. Phillips, 2005: Tropical Atlantic Influence on European Heat Waves. Journal of Climate, 18 (15), 2805-2811, doi:10.1175/JCLI3506.1.

Cheng, X., and J. M. Wallace, 1993: Cluster Analysis of the Northern Hemisphere Wintertime 500hPa Height Field: Spatial Patterns. Journal of the Atmospheric Sciences, 50 (16), 2674-2696, doi:10.1175/1520-0469(1993)050<2674:CAOTNH>2.0.CO;2.

Cleveland, W. S., 1979: Robust locally weighted regression and smoothing scatterplots. Journal of the American Statistical Association, 74 (368), 829-836, doi:10.1080/01621459.1979. 10481038. 
Croci-Maspoli, M., C. Schwierz, and H. C. Davies, 2007: Atmospheric blocking: Space-time links to the nao and pna. Climate Dynamics, 29 (7-8), 713-725, doi:10.1007/s00382-007-0259-4.

Crommelin, D. T., 2004: Observed Nondiffusive Dynamics in Large-Scale Atmospheric Flow. Journal of the Atmospheric Sciences, 61 (19), 2384-2396, doi:10.1175/1520-0469(2004) 061<2384:ONDILA>2.0.CO;2.

Dieci, L., R. D. Russell, and E. S. Van Vleck, 1997: On the computation of lyapunov exponents for continuous dynamical systems. SIAM journal on numerical analysis, 34 (1), 402-423, doi: $10.1137 / \mathrm{S} 0036142993247311$.

Falkena, S. K., J. de Wiljes, A. Weisheimer, and T. G. Shepherd, 2020: Revisiting the identification of wintertime atmospheric circulation regimes in the euro-atlantic sector. Quarterly Journal of the Royal Meteorological Society, 1-14, doi:10.1002/qj.3818.

Faranda, D., G. Masato, N. Moloney, Y. Sato, F. Daviaud, B. Dubrulle, and P. Yiou, 2016: The switching between zonal and blocked mid-latitude atmospheric circulation: a dynamical system perspective. Climate Dynamics, 47 (5-6), 1587-1599, doi:10.1007/s00382-015-2921-6.

Faranda, D., G. Messori, and P. Yiou, 2017: Dynamical proxies of north atlantic predictability and extremes. Scientific reports, 7, 41 278, doi:10.1038/srep41278.

Feldstein, S. B., 2003: The dynamics of nao teleconnection pattern growth and decay. Quarterly Journal of the Royal Meteorological Society, 129 (589), 901-924, doi:10.1256/qj.02.76.

Fereday, D., 2017: How Persistent Are North Atlantic-European Sector Weather Regimes? Journal of Climate, 30 (7), 2381-2394, doi:10.1175/JCLI-D-16-0328.1. 
Fereday, D. R., J. R. Knight, A. A. Scaife, C. K. Folland, and A. Philipp, 2008: Cluster Analysis of North Atlantic-European Circulation Types and Links with Tropical Pacific Sea Surface Temperatures. Journal of Climate, 21 (15), 3687-3703, doi:10.1175/2007JCLI1875.1.

Franzke, C., D. Crommelin, A. Fischer, and A. J. Majda, 2008: A Hidden Markov Model Perspective on Regimes and Metastability in Atmospheric Flows. Journal of Climate, 21 (8), 1740-1757, doi:10.1175/2007JCLI1751.1.

Franzke, C., I. Horenko, A. J. Majda, and R. Klein, 2009: Systematic Metastable Atmospheric Regime Identification in an AGCM. Journal of the Atmospheric Sciences, 66 (7), 1997-2012, doi:10.1175/2009JAS2939.1.

Franzke, C., S. Lee, and S. B. Feldstein, 2004: Is the North Atlantic Oscillation a Breaking Wave? Journal of the Atmospheric Sciences, 61 (2), 145-160, doi:10.1175/1520-0469(2004)061<0145: ITNAOA>2.0.CO;2.

Franzke, C. L. E., T. J. O’Kane, D. P. Monselesan, J. S. Risbey, and I. Horenko, 2015: Systematic attribution of observed southern hemisphere circulation trends to external forcing and internal variability. Nonlinear Processes in Geophysics, 22 (5), 513-525, doi:10.5194/npg-22-513-2015.

Franzke, C. L. E., T. Woolins, and O. Martius, 2011: Persistent Circulation Regimes and Preferred Regime Transitions in the North Atlantic. Journal of the Atmospheric Sciences, 68 (12), 28092825, doi:10.1175/JAS-D-11-046.1.

Frederiksen, J., 2002: Genesis of intraseasonal oscillations and equatorial waves. Journal of the Atmospheric Sciences, 59 (19), 2761-2781., doi:10.1175/1520-0469(2002)059<2761: GOIOAE $>2.0 . \mathrm{CO} ; 2$. 
Frederiksen, J., and C. Frederiksen, 1993: Monsoon disturbances, intraseasonal oscillations, teleconnection patterns, blocking, and storm tracks of the global atmosphere during January 1979: Linear theory. Journal of the Atmospheric Sciences, 50 (10), 1349-1372, doi:10.1175/ 1520-0469(1993)050<1349:MDIOTP>2.0.CO;2.

Frederiksen, J., and H. Lin, 2013: Tropical-Extratropical Interactions of Intraseasonal Oscillations. Journal of the Atmospheric Sciences, 70 (10), 3180-3197., doi:10.1175/JAS-D-12-0302.1.

Froyland, G., T. Hüls, G. P. Morriss, and T. M. Watson, 2013: Computing covariant lyapunov vectors, oseledets vectors, and dichotomy projectors: A comparative numerical study. Physica D: Nonlinear Phenomena, 247 (1), 18-39, doi:10.1016/j.physd.2012.12.005.

Froyland, G., S. Lloyd, and A. Quas, 2010: Coherent structures and isolated spectrum for perron-frobenius cocycles. Ergodic Theory and Dynamical Systems, 30 (3), 729-756, doi: $10.1017 / \mathrm{S} 0143385709000339$.

Ginelli, F., P. Poggi, A. Turchi, H. Chaté, R. Livi, and A. Politi, 2007: Characterizing dynamics with covariant lyapunov vectors. Phys. Rev. Lett., 99, 130 601, doi:10.1103/PhysRevLett.99.130601.

Hanna, E., T. E. Cropper, P. D. Jones, A. A. Scaife, and R. Allan, 2015: Recent seasonal asymmetric changes in the nao (a marked summer decline and increased winter variability) and associated changes in the ao and greenland blocking index. International Journal of Climatology, 35 (9), 2540-2554, doi:10.1002/joc.4157.

Hannachi, A., and B. Legras, 1995: Simulated annealing and weather regimes classification. Tellus A, 47 (5), 955-973, doi:10.1034/j.1600-0870.1995.00203.x.

Harries, D., and T. J. O’Kane, 2020: Applications of matrix factorization methods to climate data. Nonlinear Processes in Geophysics, 27 (3), 453-471, doi:10.5194/npg-27-453-2020. 
Horenko, I., 2009: On robust estimation of low-frequency variability trends in discrete markovian sequences of atmospheric circulation patterns. Journal of the atmospheric sciences, 66 (7), 2059-2072, doi:10.1175/2008JAS2959.1.

Horenko, I., 2010a: On clustering of non-stationary meteorological time series. Dynamics of Atmospheres and Oceans, 49 (2-3), 164-187, doi:10.1016/j.dynatmoce.2009.04.003.

Horenko, I., 2010b: On the identification of nonstationary factor models and their application to atmospheric data analysis. Journal of the Atmospheric Sciences, 67 (5), 1559-1574, doi: 10.1175/2010JAS3271.1

Hunter, J. D., 2007: Matplotlib: A 2d graphics environment. Computing in Science \& Engineering, 9 (3), 90-95, doi:10.1109/MCSE.2007.55.

Hurrell, J. W., 1995: Decadal trends in the north atlantic oscillation: Regional temperatures and precipitation. Science, 269 (5224), 676-679, doi:10.1126/science.269.5224.676.

Hurrell, J. W., Y. Kushnir, G. Ottersen, and M. Visbeck, 2013: An Overview of the North Atlantic Oscillation, 1-35. American Geophysical Union (AGU), doi:10.1029/134GM01.

Huth, R., C. Beck, A. Philipp, M. Demuzere, Z. Ustrnul, M. Cahynová, J. Kyselý, and O. E. Tveito, 2008: Classifications of atmospheric circulation patterns. Annals of the New York Academy of Sciences, 1146 (1), 105-152, doi:10.1196/annals.1446.019.

Kalnay, E., and Coauthors, 1996: The ncep/ncar 40-year reanalysis project. Bulletin of the American meteorological Society, 77 (3), 437-472, doi:10.1175/1520-0477(1996)077\%3C0437: TNYRP\%3E2.0.CO;2. 
Kidson, J. W., 2000: An analysis of new zealand synoptic types and their use in defining weather regimes. International Journal of Climatology, 20 (3), 299-316, doi:10.1002/(SICI) 1097-0088(20000315)20:3<299::AID-JOC474>3.0.CO;2-B.

Kimoto, M., and M. Ghil, 1993a: Multiple Flow Regimes in the Northern Hemisphere Winter. Part I: Methodology and Hemispheric Regimes. Journal of the Atmospheric Sciences, 50 (16), 2625-2644, doi:10.1175/1520-0469(1993)050<2625:MFRITN>2.0.CO;2.

Kimoto, M., and M. Ghil, 1993b: Multiple Flow Regimes in the Northern Hemisphere Winter. Part II: Sectorial Regimes and Preferred Transitions. Journal of the Atmospheric Sciences, 50 (16), 2645-2673, doi:10.1175/1520-0469(1993)050<2645:MFRITN>2.0.CO;2.

Kuptsov, P. V., and S. P. Kuznetsov, 2018: Lyapunov analysis of strange pseudohyperbolic attractors: angles between tangent subspaces, local volume expansion and contraction. Regular and Chaotic Dynamics, 23 (7-8), 908-932, doi:10.1134/S1560354718070079.

Kuptsov, P. V., and U. Parlitz, 2012: Theory and computation of covariant lyapunov vectors. Journal of nonlinear science, 22 (5), 727-762, doi:10.1007/s00332-012-9126-5.

Legras, B., and M. Ghil, 1985: Persistent Anomalies, Blocking and Variations in Atmospheric Predictability. Journal of the Atmospheric Sciences, 42 (5), 433-471, doi:10.1175/1520-0469(1985) 042<0433:PABAVI>2.0.CO;2.

Lin, H., G. Brunet, and J. Derome, 2018: An Observed Connection between the North Atlantic Oscillation and the Madden-Julian (O)scillation. Journal of Climate, 22 (2), 364-380., doi: 10.1175/2008JCLI2515.1.

Lucarini, V., and A. Gritsun, 2020: A new mathematical framework for atmospheric blocking events. Climate Dynamics, 54 (1-2), 575-598, doi:10.1007/s00382-019-05018-2. 
Luo, D., and J. Cha, 2012: The North Atlantic Oscillation and the North Atlantic Jet Variability: Precursors to NAO Regimes and Transitions. Journal of the Atmospheric Sciences, 69 (12), 3763-3787, doi:10.1175/JAS-D-12-098.1.

Luo, D., T. Gong, and Y. Diao, 2007a: Dynamics of Eddy-Driven Low-Frequency Dipole Modes. Part III: Meridional Displacement of Westerly Jet Anomalies during Two Phases of NAO. Journal of the Atmospheric Sciences, 64 (9), 3232-3248, doi:10.1175/JAS3998.1.

Luo, D., T. Gong, and A. R. Lupo, 2007b: Dynamics of Eddy-Driven Low-Frequency Dipole Modes. Part II: Free Mode Characteristics of NAO and Diagnostic Study. Journal of the Atmospheric Sciences, 64 (1), 29-51, doi:10.1175/JAS3820.1.

Luo, D., A. R. Lupo, and H. Wan, 2007c: Dynamics of Eddy-Driven Low-Frequency Dipole Modes. Part I: A Simple Model of North Atlantic Oscillations. Journal of the Atmospheric Sciences, 64 (1), 3-28, doi:10.1175/JAS3818.1.

Majda, A. J., C. L. Franzke, A. Fischer, and D. T. Crommelin, 2006: Distinct metastable atmospheric regimes despite nearly gaussian statistics: A paradigm model. Proceedings of the National Academy of Sciences, 103 (22), 8309-8314, doi:10.1073/pnas.0602641103.

Metzner, P., L. Putzig, and I. Horenko, 2012: Analysis of persistent nonstationary time series and applications. Communications in Applied Mathematics and Computational Science, 7 (2), 175-229, doi:10.2140/camcos.2012.7.175.

Michelangeli, P.-A., R. Vautard, and B. Legras, 1995: Weather Regimes: Recurrence and Quasi Stationarity. Journal of the Atmospheric Sciences, 52 (8), 1237-1256, doi:10.1175/ 1520-0469(1995)052<1237:WRRAQS>2.0.CO;2. 
Mo, K., and M. Ghil, 1988: Cluster analysis of multiple planetary flow regimes. Journal of Geophysical Research: Atmospheres, 93 (D9), 10 927-10 952, doi:10.1029/JD093iD09p10927.

Molteni, F., S. Tibaldi, and T. N. Palmer, 1990: Regimes in the wintertime circulation over northern extratropics. i: Observational evidence. Quarterly Journal of the Royal Meteorological Society, 116 (491), 31-67, doi:10.1002/qj.49711649103.

Neal, R., D. Fereday, R. Crocker, and R. E. Comer, 2016: A flexible approach to defining weather patterns and their application in weather forecasting over europe. Meteorological Applications, 23 (3), 389-400, doi:10.1002/met.1563.

Norwood, A., E. Kalnay, K. Ide, S.-C. Yang, and C. Wolfe, 2013: Lyapunov, singular and bred vectors in a multi-scale system: an empirical exploration of vectors related to instabilities. Journal of Physics A: Mathematical and Theoretical, 46 (25), 254 021, doi:10.1088/1751-8113/ $46 / 25 / 254021$

O’Kane, T. J., R. J. Matear, M. A. Chamberlain, J. S. Risbey, B. M. Sloyan, and I. Horenko, 2013a: Decadal variability in an ogcm southern ocean: Intrinsic modes, forced modes and metastable states. Ocean Modelling, 69, 1-21, doi:10.1016/j.ocemod.2013.04.009.

O’Kane, T. J., D. P. Monselesan, J. S. Risbey, I. Horenko, and C. L. E. Franzke, 2017: On memory, dimension, and atmospheric teleconnections. Mathematics of Climate and Weather Forecasting, 3, 1-27, doi:10.1515/mcwf-2017-0001.

O'Kane, T. J., J. S. Risbey, C. Franzke, I. Horenko, and D. P. Monselesan, 2013b: Changes in the metastability of the midlatitude southern hemisphere circulation and the utility of nonstationary cluster analysis and split-flow blocking indices as diagnostic tools. Journal of the atmospheric sciences, 70 (3), 824-842, doi:10.1175/JAS-D-12-028.1. 
O’ Kane, T. J., J. S. Risbey, D. P. Monselesan, I. Horenko, and C. L. Franzke, 2016: On the dynamics of persistent states and their secular trends in the waveguides of the southern hemisphere troposphere. Climate Dynamics, 46 (11-12), 3567-3597, doi:10.1007/s00382-015-2786-8.

Oseledets, V. I., 1968: A multiplicative ergodic theorem. characteristic ljapunov, exponents of dynamical systems. Trudy Moskovskogo Matematicheskogo Obshchestva, 19, 179-210.

Pazó, D., I. G. Szendro, J. M. López, and M. A. Rodríguez, 2008: Structure of characteristic lyapunov vectors in spatiotemporal chaos. Phys. Rev. E, 78, 016 209, doi:10.1103/PhysRevE.78. 016209.

Pedregosa, F., and Coauthors, 2011: Scikit-learn: Machine learning in Python. Journal of Machine Learning Research, 12, 2825-2830.

Plaut, G., and E. Simmonet, 2001: Large-scale circulation classification, weather regimes, and local climate over France, the Alps and Western Europe. Climate Research, 17, 303 - 324, doi:10.3354/cr017303.

Pohl, B., and N. Fauchereau, 2012: The Southern Annular Mode Seen through Weather Regimes. Journal of Climate, 25 (9), 3336-3354, doi:10.1175/JCLI-D-11-00160.1.

Quinn, C., T. J. O’Kane, and V. Kitsios, 2020: Application of a local attractor dimension to reduced space strongly coupled data assimilation for chaotic multiscale systems. Nonlinear Processes in Geophysics, 27 (1), 51-74, doi:10.5194/npg-27-51-2020.

Renwick, J. A., 2005: Persistent Positive Anomalies in the Southern Hemisphere Circulation. Monthly Weather Review, 133 (4), 977-988, doi:10.1175/MWR2900.1. 
Risbey, J. S., T. J. O’Kane, D. P. Monselesan, C. L. E. Franzke, and I. Horenko, 2018: On the dynamics of austral heat waves. Journal of Geophysical Research: Atmospheres, 123 (1), 38-57, doi:10.1002/2017JD027222.

Risbey, J. S., T. J. O’Kane, D. P. Monselesan, C. Franzke, and I. Horenko, 2015: Metastability of northern hemisphere teleconnection modes. Journal of the Atmospheric Sciences, 72 (1), 35-54, doi:10.1175/JAS-D-14-0020.1.

Ruelle, D., 1979: Ergodic theory of differentiable dynamical systems. Publications Mathématiques de l'Institut des Hautes Études Scientifiques, 50 (1), 27-58, doi:10.1007/BF02684768.

Schubert, S., and V. Lucarini, 2015: Covariant lyapunov vectors of a quasi-geostrophic baroclinic model: analysis of instabilities and feedbacks. Quarterly Journal of the Royal Meteorological Society, 141 (693), 3040-3055, doi:10.1002/qj.2588.

Schubert, S., and V. Lucarini, 2016: Dynamical analysis of blocking events: spatial and temporal fluctuations of covariant lyapunov vectors. Quarterly Journal of the Royal Meteorological Society, 142 (698), 2143-2158, doi:10.1002/qj.2808.

Shabbar, A., J. Huang, and K. Higuchi, 2001: The relationship between the wintertime north atlantic oscillation and blocking episodes in the north atlantic. International Journal of Climatology, 21 (3), 355-369, doi:10.1002/joc.612.

Sharafi, N., M. Timme, and S. Hallerberg, 2017: Critical transitions and perturbation growth directions. Physical Review E, 96 (3), 032 220, doi:10.1103/PhysRevE.96.032220.

Smyth, P., K. Ide, and M. Ghil, 1999: Multiple Regimes in Northern Hemisphere Height Fields via MixtureModel Clustering*. Journal of the Atmospheric Sciences, 56 (21), 3704-3723, doi: 10.1175/1520-0469(1999)056<3704:MRINHH>2.0.CO;2. 
Stan, C., and D. M. Straus, 2007: Is Blocking a Circulation Regime? Monthly Weather Review, 135 (6), 2406-2413, doi:10.1175/MWR3410.1.

Stephenson, D. B., V. Pavan, and R. Bojariu, 2000: Is the north atlantic oscillation a random walk? International Journal of Climatology: A Journal of the Royal Meteorological Society, 20 (1), 1-18, doi:10.1002/(SICI)1097-0088(200001)20:1\%3C1::AID-JOC456\%3E3.0.CO;2-P.

Stone, R. C., 1989: Weather types at brisbane, queensland: an example of the use of principal components and cluster analysis. International Journal of Climatology, 9 (1), 3-32, doi:10. 1002/joc.3370090103.

Straus, D. M., S. Corti, and F. Molteni, 2007: Circulation Regimes: Chaotic Variability versus SST-Forced Predictability. Journal of Climate, 20 (10), 2251-2272, doi:10.1175/JCLI4070.1.

Straus, D. M., F. Molteni, and S. Corti, 2017: Atmospheric regimes: The link between weather and the large-scale circulation. Nonlinear and Stochastic Climate Dynamics, C. L. E. Franzke, and T. J. O'Kane, Eds., Cambridge University Press, chap. 4, 105-135, doi:10.1017/9781316339251. 005.

Tantet, A., F. R. van der Burgt, and H. A. Dijkstra, 2015: An early warning indicator for atmospheric blocking events using transfer operators. Chaos: An Interdisciplinary Journal of Nonlinear Science, 25 (3), 036 406, doi:10.1063/1.4908174.

Trevisan, A., and F. Pancotti, 1998: Periodic orbits, lyapunov vectors, and singular vectors in the lorenz system. Journal of the atmospheric sciences, 55 (3), 390-398, doi: 10.1175/1520-0469(1998)055\%3C0390:POLVAS\%3E2.0.CO;2. 
Vallis, G. K., E. P. Gerber, P. J. Kushner, and B. A. Cash, 2004: A Mechanism and Simple Dynamical Model of the North Atlantic Oscillation and Annular Modes. Journal of the Atmospheric Sciences, 61 (3), 264-280, doi:10.1175/1520-0469(2004)061<0264:AMASDM>2.0.CO;2.

Vautard, R., 1990: Multiple Weather Regimes over the North Atlantic: Analysis of Precursors and Successors. Monthly Weather Review, 118 (10), 2056-2081, doi:10.1175/1520-0493(1990) 118<2056:MWROTN>2.0.CO;2.

Vercauteren, N., and R. Klein, 2015: A Clustering Method to Characterize Intermittent Bursts of Turbulence and Interaction with Submesomotions in the Stable Boundary Layer. Journal of the Atmospheric Sciences, 72 (4), 1504-1517, doi:10.1175/JAS-D-14-0115.1.

Vercauteren, N., L. Mahrt, and R. Klein, 2016: Investigation of interactions between scales of motion in the stable boundary layer. Quarterly Journal of the Royal Meteorological Society, 142 (699), 2424-2433, doi:10.1002/qj.2835.

Visbeck, M. H., J. W. Hurrell, L. Polvani, and H. M. Cullen, 2001: The north atlantic oscillation: past, present, and future. Proceedings of the National Academy of Sciences, 98 (23), 12 87612 877, doi:10.1073/pnas.231391598.

Wolfe, C. L., and R. M. Samelson, 2007: An efficient method for recovering lyapunov vectors from singular vectors. Tellus A: Dynamic Meteorology and Oceanography, 59 (3), 355-366, doi:10.1111/j.1600-0870.2007.00234.x.

Woollings, T., B. Hoskins, M. Blackburn, and P. Berrisford, 2008: A new rossby wave-breaking interpretation of the north atlantic oscillation. Journal of the Atmospheric Sciences, 65 (2), 609-626, doi:10.1175/2007JAS2347.1. 


\section{LIST OF TABLES}

Table 1. Summary statistics for the run lengths (in days) of consecutive days assigned to each state for the model with $K=3, m=3$ days, and $p=5$ days.

Table 2. Counts of number of transitions and the total number of days assigned to each state, stratified by season. Transitions are assigned to the season corresponding to the last day in the initial state. Note that $m_{\max }=5$ days are held out as presample values from the full record of $T=14610$ days, yielding a total fit

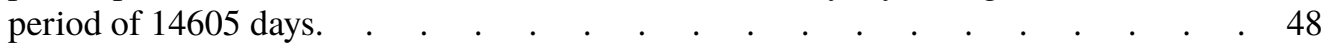

Table 3. Probabilities associated with the occurrence of positive FTEs for short and long push forward steps. Note that the total number of days for which the CLVs are calculated depends on the push forward step $\left(T_{M}=14605-2 M\right.$ days $)$.

Table 4. Average $\operatorname{dim}_{K Y}(t)$ measure by state. The first column is averaged over all days associated with each state. The second column averages over the associated days using a 5-day filter, namely only taking the values from time instances where the 2 days before and the 2 days after are also associated with the same state. . . . . . . . . . . . . . . . . . . . . . . 50

Table 5. Characteristics of unstable patterns associated with transitions to and from persistent states (shown in Fig. 6). The day column refers to the day in the end state after the transition. . . . . . . . . . . . . . . . . 5 
TABLE 1. Summary statistics for the run lengths (in days) of consecutive days assigned to each state for the model with $K=3, m=3$ days, and $p=5$ days.

\begin{tabular}{cccccc|c}
\hline \hline & & DJF & MAM & JJA & SON & ALL \\
\hline AR & Mean & 2.8 & 2.4 & 2.5 & 2.9 & 2.7 \\
& Max. & 21 & 13 & 15 & 18 & 21 \\
\hline & Min. & 1 & 1 & 1 & 1 & 1 \\
NAO $^{-}$ & Mean & 2.5 & 4.3 & 9.3 & 3.2 & 4.7 \\
& Max. & 21 & 38 & 63 & 29 & 63 \\
\hline & Min. & 1 & 1 & 1 & 1 & 1 \\
NAO $^{+}$ & Mean & 3.3 & 2.4 & 2.2 & 2.4 & 2.7 \\
& Max. & 26 & 11 & 10 & 12 & 26 \\
\hline
\end{tabular}


TABLE 2. Counts of number of transitions and the total number of days assigned to each state, stratified by season. Transitions are assigned to the season corresponding to the last day in the initial state. Note that $m_{\max }=5$ days are held out as presample values from the full record of $T=14610$ days, yielding a total fit period of 14605 days.

\begin{tabular}{|c|c|c|c|c|c|c|}
\hline & & DJF & MAM & JJA & SON & ALL \\
\hline \multirow{7}{*}{ Transitions } & $\mathrm{AR}$ to $\mathrm{NAO}^{-}$ & 136 & 213 & 168 & 234 & 751 \\
\hline & AR to $\mathrm{NAO}^{+}$ & 310 & 147 & 44 & 209 & 710 \\
\hline & $\mathrm{NAO}^{-}$to $\mathrm{AR}$ & 118 & 197 & 176 & 219 & 710 \\
\hline & $\mathrm{NAO}^{-}$to $\mathrm{NAO}^{+}$ & 177 & 214 & 131 & 228 & 750 \\
\hline & $\mathrm{NAO}^{+}$to $\mathrm{AR}$ & 327 & 153 & 42 & 228 & 750 \\
\hline & $\mathrm{NAO}^{+}$to $\mathrm{NAO}^{-}$ & 163 & 218 & 129 & 200 & 710 \\
\hline & Any & 1232 & 1142 & 690 & 1318 & 4381 \\
\hline \multirow{4}{*}{ Days assigned to } & $\mathrm{AR}$ & 1229 & 859 & 539 & 1274 & 3901 \\
\hline & $\mathrm{NAO}^{-}$ & 725 & 1974 & 2771 & 1326 & 6796 \\
\hline & $\mathrm{NAO}^{+}$ & 1651 & 847 & 370 & 1040 & 3908 \\
\hline & Any & 3605 & 3680 & 3680 & 3640 & 14605 \\
\hline
\end{tabular}


TABLE 3. Probabilities associated with the occurrence of positive FTEs for short and long push forward steps. Note that the total number of days for which the CLVs are calculated depends on the push forward step ${ }_{962}\left(T_{M}=14605-2 M\right.$ days $)$.

\begin{tabular}{cccccc}
\hline \hline & & $M=3$ & $M=10$ & $M=30$ & $M=50$ \\
\hline \multirow{2}{*}{$\mathrm{P}\left(\operatorname{dim}_{K Y}>0\right)$} & $\mathrm{NAO}^{-}$ & 0.992 & 0.002 & 0.001 & 0 \\
& $\mathrm{NAO}^{+}$ & 0.624 & 0.007 & 0.001 & 0.001 \\
\cline { 2 - 6 } & Any & 0.733 & 0.004 & 0.001 & 0.001 \\
\hline
\end{tabular}


TABLE 4. Average $\operatorname{dim}_{K Y}(t)$ measure by state. The first column is averaged over all days associated with each state. The second column averages over the associated days using a 5-day filter, namely only taking the values

from time instances where the 2 days before and the 2 days after are also associated with the same state.

\begin{tabular}{ccc}
\hline \hline & no filter & 5-day filter \\
\hline $\mathrm{AR}$ & 0.84 & 0 \\
$\mathrm{NAO}^{-}$ & 2.55 & 2.98 \\
$\mathrm{NAO}^{+}$ & 1.16 & 1.28 \\
\hline
\end{tabular}



in FIG. 6). The day column refers to the day in the end state after the transition.

\begin{tabular}{ccccc}
\hline \hline Pattern & Transition & day & CLV & FTE \\
\hline & $\mathrm{NAO}^{-}$to $\mathrm{AR}$ & 1 & 1 & 0.029 \\
$\mathrm{~A}$ & $\mathrm{NAO}^{-}$to $\mathrm{NAO}^{+}$ & 1 & 1 & 0.058 \\
& $\mathrm{NAO}^{-}$to $\mathrm{NAO}^{+}$ & 2 & 2 & 0.012 \\
\hline $\mathrm{B}$ & $\mathrm{NAO}^{-}$to $\mathrm{NAO}^{+}$ & 1 & 2 & 0.023 \\
\hline $\mathrm{C}$ & $\mathrm{NAO}^{+}$to $\mathrm{AR}^{-}$ & 2 & 2 & 0.017 \\
\hline & $\mathrm{NAO}^{-}$to $\mathrm{NAO}^{+}$ & 2 & 1 & 0.031 \\
$\mathrm{D}$ & $\mathrm{NAO}^{+}$to $\mathrm{NAO}^{-}$ & 1 & 1 & 0.027 \\
\hline
\end{tabular}




\section{LIST OF FIGURES}

Fig. 1. Mean test set reconstruction error as a function of typical state length $p$ (main figure), and zoom to the region containing the model with minimal mean reconstruction RMSE (inset). Note that $p=0$ corresponds to no persistence constraint imposed (i.e., $C_{T} \rightarrow \infty$ ). Error bars show the approximate one standard error ranges, and for clarity models with the same VAR order $m$ are offset in the $x$-direction. The minimal mean reconstruction RMSE occurs for $K=3, m=3$ days, and $p=5$ days.

Fig. 2. Composites of $Z_{g 500 \mathrm{hPa}}^{\prime}$ in each of the FEM-BV-VAR states for the model with $K=3, m=3$ days, and $p=5$ days. Shading indicates regions for which the composite value lies outside of the interval containing $100(1-\alpha)=99 \%$ of 1000 bootstrap samples drawn assuming the number of samples assigned to each state is fixed.

Fig. 3. Model $\mathrm{NAO}^{-}$state residency percent compared to residency percent for occurrences of a negative CPC NAO index value using a sliding window of one year (top panel) and yearly average with LOWESS smoothing (bottom panel). Note that the colors in the bottom panel correspond to the legend in the top panel.

Fig. 4. Statistics of the finite-time growth rates for the leading 10 CLVs computed using varying push forward steps $(M=3,10,30,50)$ compared to their asymptotic growth rates.

Fig. 5. Physical projections of unstable MSVs (computed for $M=3$ ) in persistent states (i.e. having resided in the same state at least two days prior and two days following). We take the leading 20 directions of growth in the MSV and project onto the corresponding 20 EOFs (FIG. A1). All projections use the same color bar scale. As the MSVs and EOFs are unit normalized and the EOFs are orthogonal, the projections shown here are also unit normalized.

Fig. 6. Physical projections of unstable MSVs (computed for $M=3$ ) at transitions associated with persistent states (i.e. residency greater than 4 days in the state before and after the transition). The labels for each transition correspond to those discussed in TABLE 5. All projections use the same color bar scale. As the MSVs and EOFs are unit normalized and the EOFs are orthogonal, the projections shown here are also unit normalized.

Fig. 7. Transient behavior of the leading CLV alignments $\left(\theta_{1,2}, \theta_{2,3}\right.$, and $\left.\theta_{1,3}\right)$, growth rates $\left(\Lambda_{1}, \Lambda_{2}\right.$, and $\Lambda_{3}$ ), and finite-time dimension for two different but representative time segments using push forward $M=3$. We also plot the state indicators to compare to transitions.

Fig. 8. Box and whisker plots of $\theta_{1,2}, \theta_{2,3}$, and $\theta_{1,3}$ around each transition with Day 0 indicating the last day in the previous state and Day 1 the first day in the following state. Diamonds indicate outlier values. The transitions have been filtered to only include those associated with residencies longer than 4 days both before and after the transition.

Fig. 9. Collective trajectories of $\theta_{1,2}, \theta_{2,3}$, and $\theta_{1,3}$ separated by specific transition. The transitions have been filtered as in Fig. 8 .

Fig. 10. (a) Alignment of the leading two CLVs for different push forward steps. From top to bottom: raw $\mathrm{NAO}^{-}$signal, $M=3, M=10, M=30, M=50$. (b) Power spectral density of the corresponding alignment time series. Red dots (crosses) indicate peaks that are 2 (3) standard deviations away from neighboring measures.

Fig. 11. Alignment of the leading two CLVs for push forward step $M=50$ compared to transition index calculated from Eq. (19). 
Fig. 12. Comparison of average alignment $\left(\overline{\theta_{i, j}}\right)$ of leading CLVs by season for push forward $M=50$. We see the strong alignment emerging in the JJA $\overline{\theta_{1,2}}$, and a weak alignment in both SON and DJF. Additionally we observe some seasonality in $\overline{\theta_{2,3}}$ and $\overline{\theta_{3,4}}$, with both peaking in DJF. . 65

Fig. A1. Leading $20 \mathrm{EOFs}$ of daily $500 \mathrm{hPa}$ geopotential height anomalies in the North Atlantic sector $\left(20^{\circ} \mathrm{N}-90^{\circ} \mathrm{N}\right.$ and $\left.110^{\circ} \mathrm{W}-0^{\circ} \mathrm{E}\right)$ of the daily NCEP/NCAR reanalysis data (Kalnay et al. 1996). All EOFs are unit normalized and use the same color scale shown at the bottom.

Fig. D1. Alignment, FTEs, and unit normalized physical projections of the leading MSVs throughout the transition from a persistent Atlantic Ridge state to a persistent negative NAO state. . . . . . 67

Fig. D2. Same as Fig. D1, but for the Atlantic Ridge to positive NAO transition. . . . . . . . . 68

Fig. D3. Same as Fig. D1, but for the negative NAO to Atlantic Ridge transition. . . . . . . . . 69

Fig. D4. Same as FIg. D1, but for the negative NAO to positive NAO transition. . . . . . . . 70

Fig. D5. Same as FIg. D1, but for the positive NAO to Atlantic Ridge transition. . . . . . . . . . 71

Fig. D6. Same as Fig. D1, but for the positive NAO to negative NAO transition. . . . . . . . . 72 


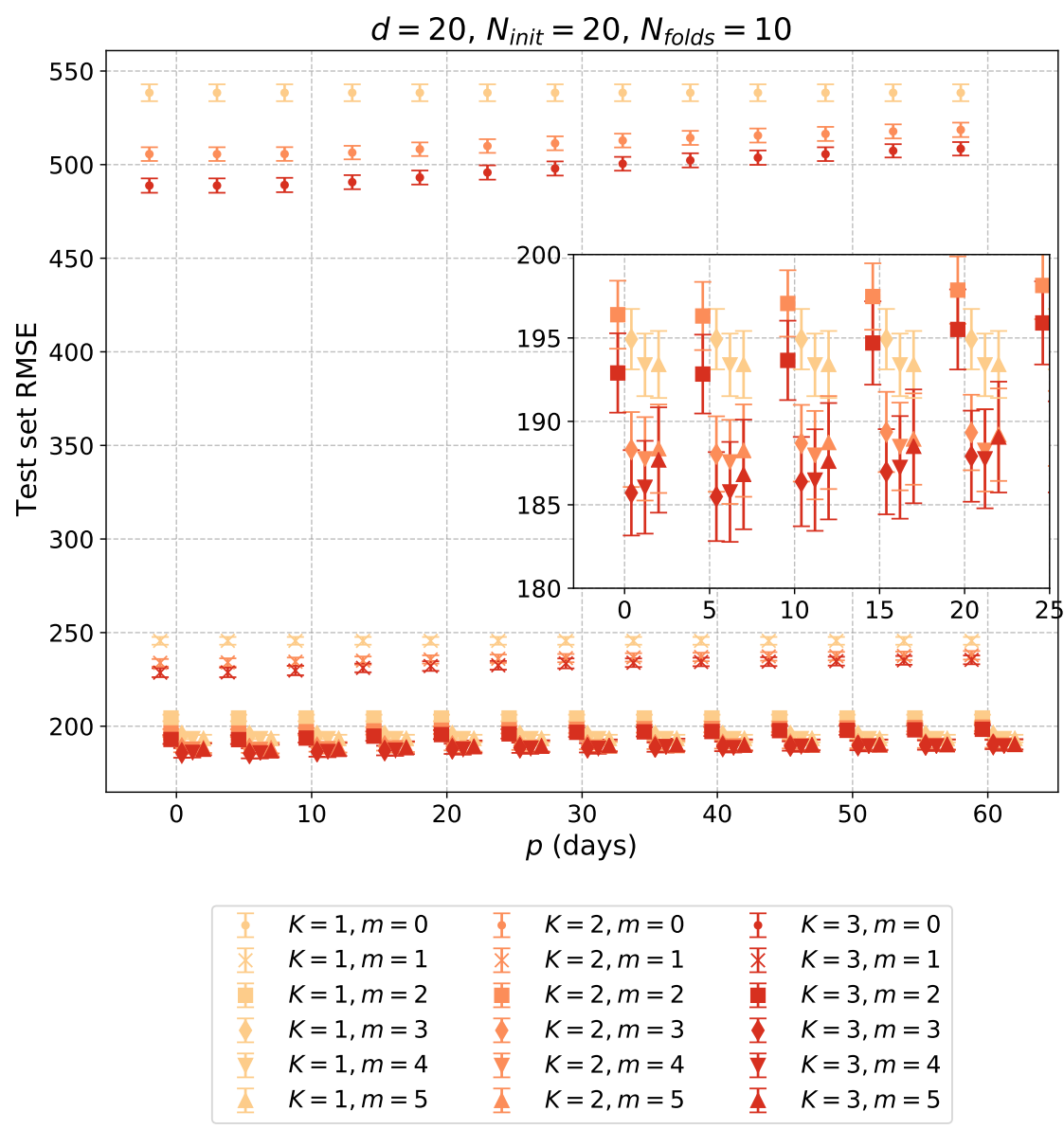

FIG. 1. Mean test set reconstruction error as a function of typical state length $p$ (main figure), and zoom to the region containing the model with minimal mean reconstruction RMSE (inset). Note that $p=0$ corresponds to no persistence constraint imposed (i.e., $C_{T} \rightarrow \infty$ ). Error bars show the approximate one standard error ranges, and for clarity models with the same VAR order $m$ are offset in the $x$-direction. The minimal mean reconstruction RMSE occurs for $K=3, m=3$ days, and $p=5$ days. 

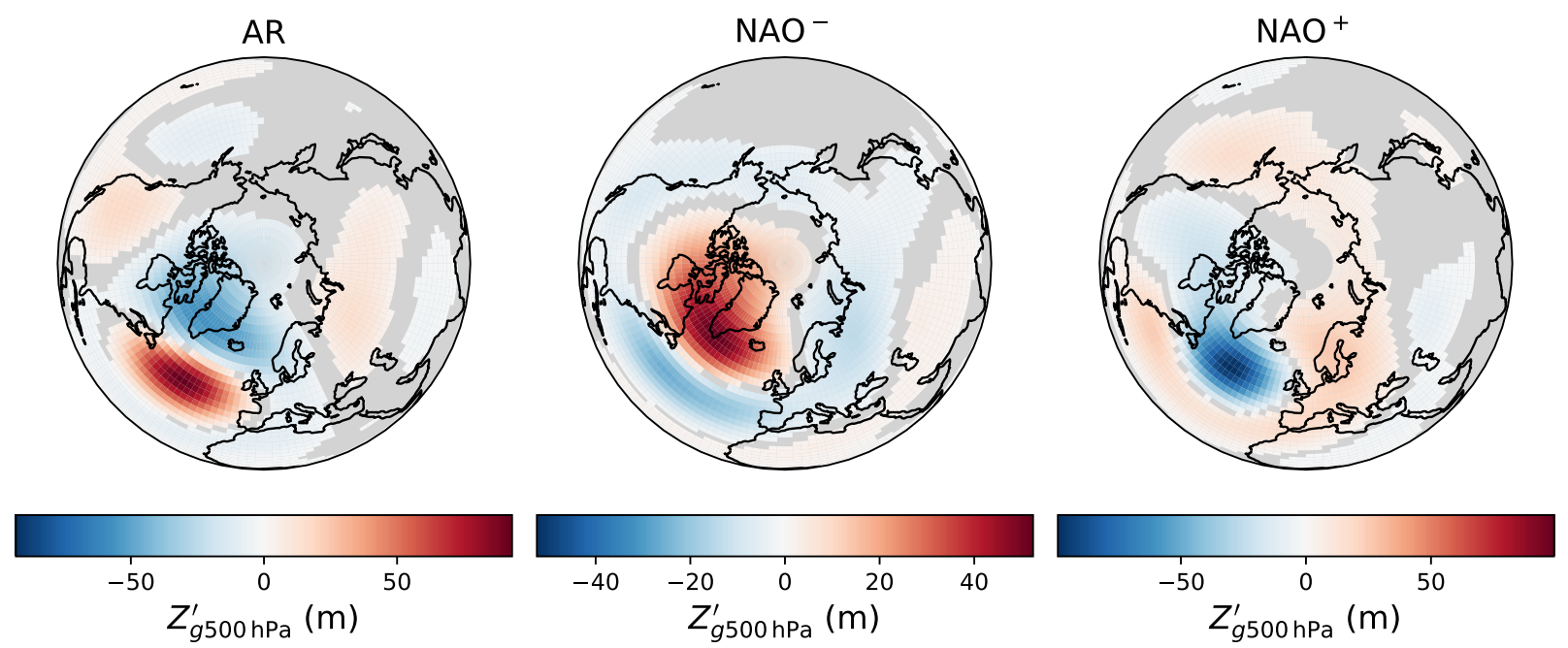

FIG. 2. Composites of $Z_{g 500 \mathrm{hPa}}^{\prime}$ in each of the FEM-BV-VAR states for the model with $K=3, m=3$ days, and $p=5$ days. Shading indicates regions for which the composite value lies outside of the interval containing $100(1-\alpha)=99 \%$ of 1000 bootstrap samples drawn assuming the number of samples assigned to each state is fixed. 


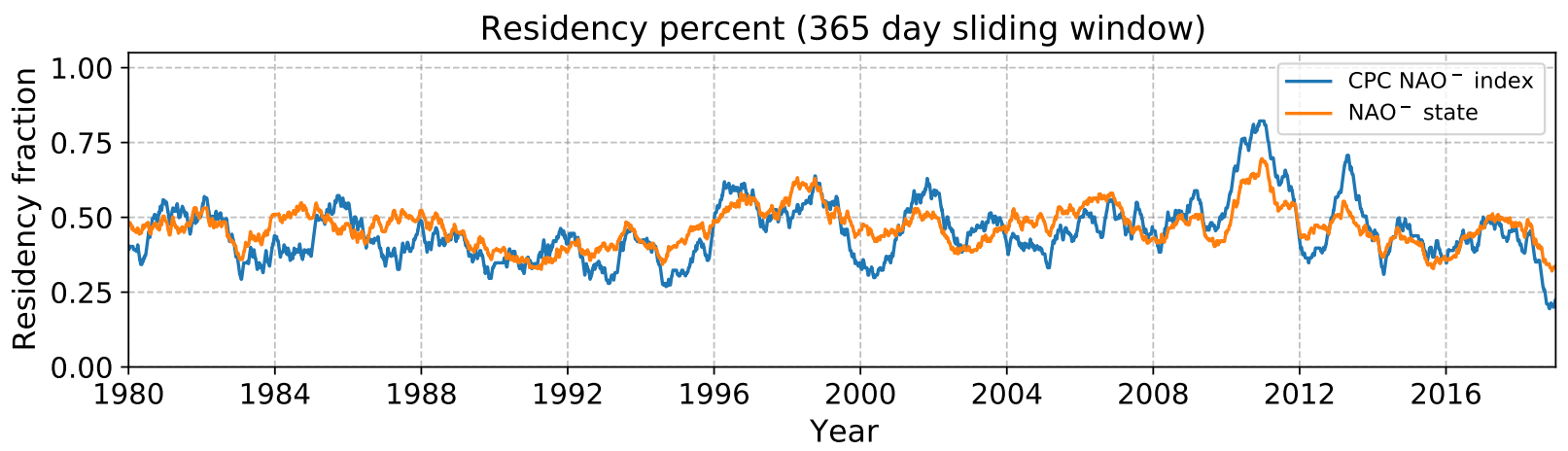

Residency percent (yearly average and LOWESS)

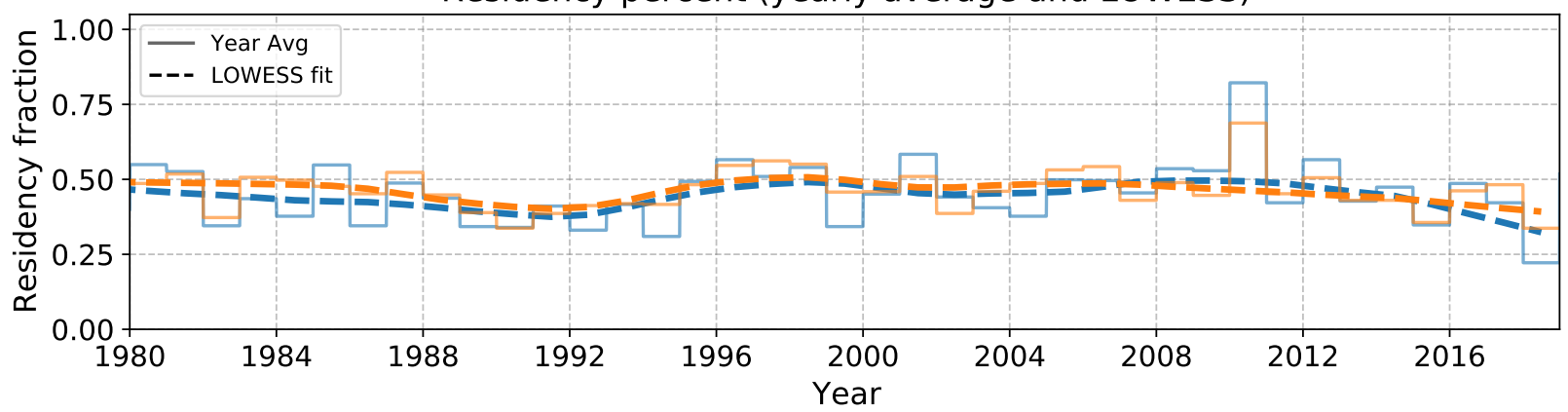

FIg. 3. Model $\mathrm{NAO}^{-}$state residency percent compared to residency percent for occurrences of a negative CPC NAO index value using a sliding window of one year (top panel) and yearly average with LOWESS smoothing (bottom panel). Note that the colors in the bottom panel correspond to the legend in the top panel. 

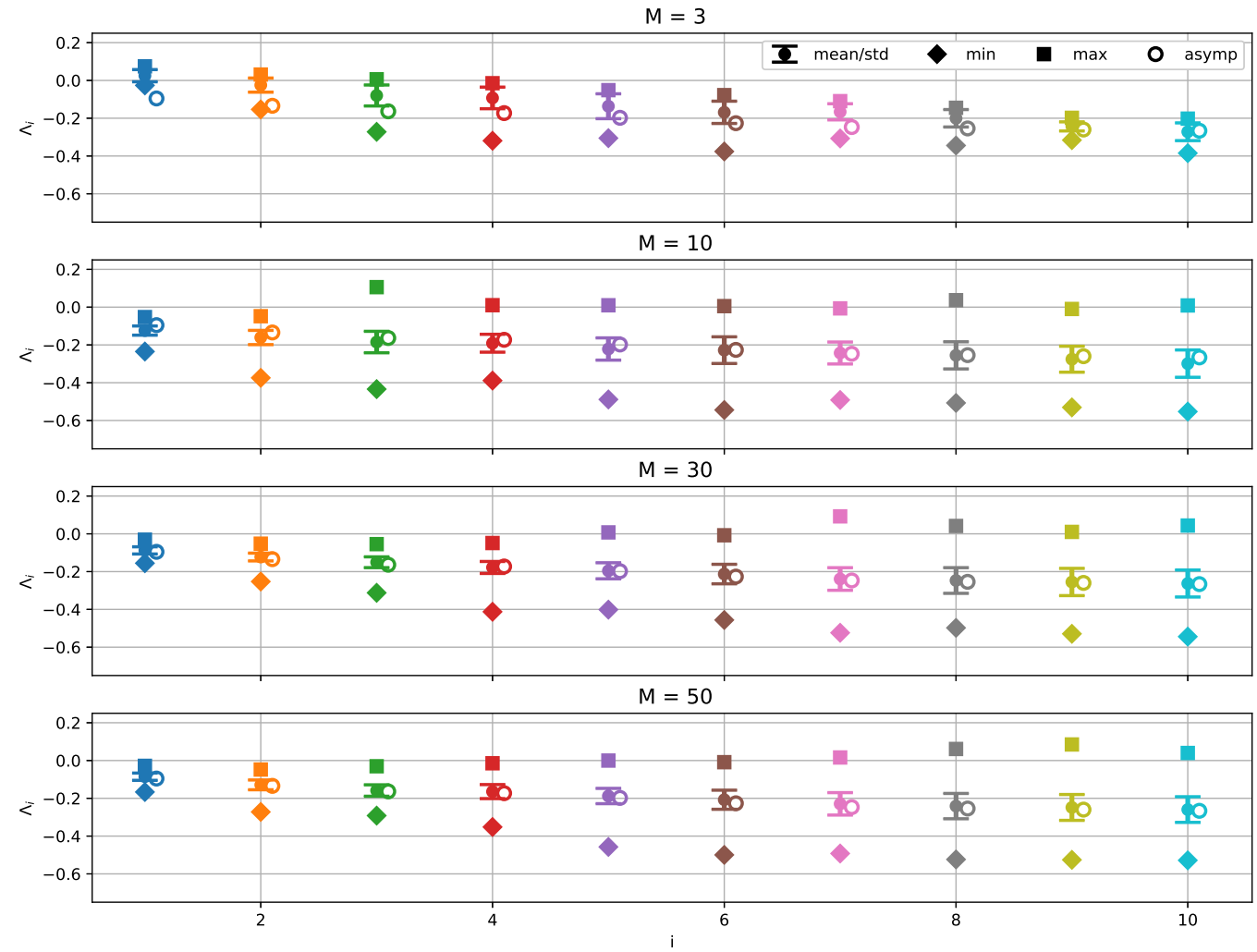

FIG. 4. Statistics of the finite-time growth rates for the leading 10 CLVs computed using varying push forward steps $(M=3,10,30,50)$ compared to their asymptotic growth rates. 


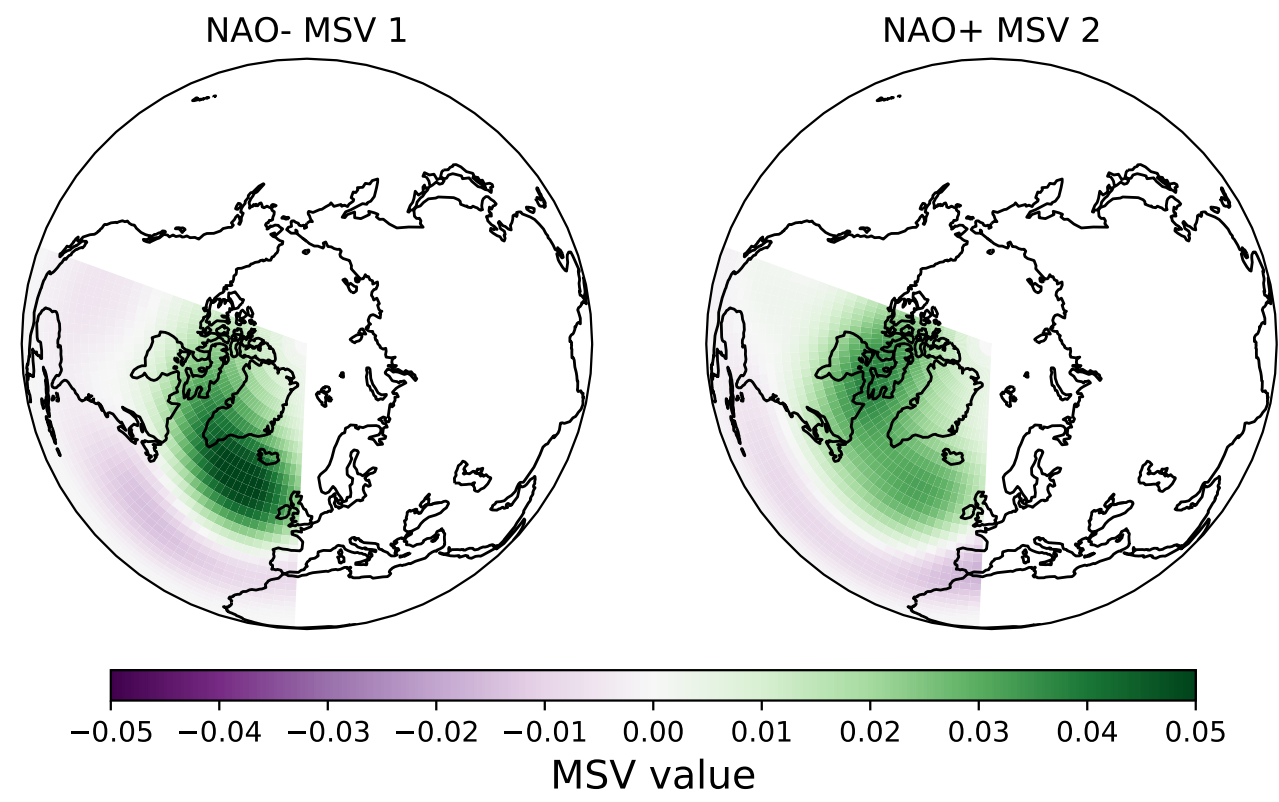

FIg. 5. Physical projections of unstable MSVs (computed for $M=3$ ) in persistent states (i.e. having resided in the same state at least two days prior and two days following). We take the leading 20 directions of growth in

As the MSVs and EOFs are unit normalized and the EOFs are orthogonal, the projections shown here are also unit normalized. 


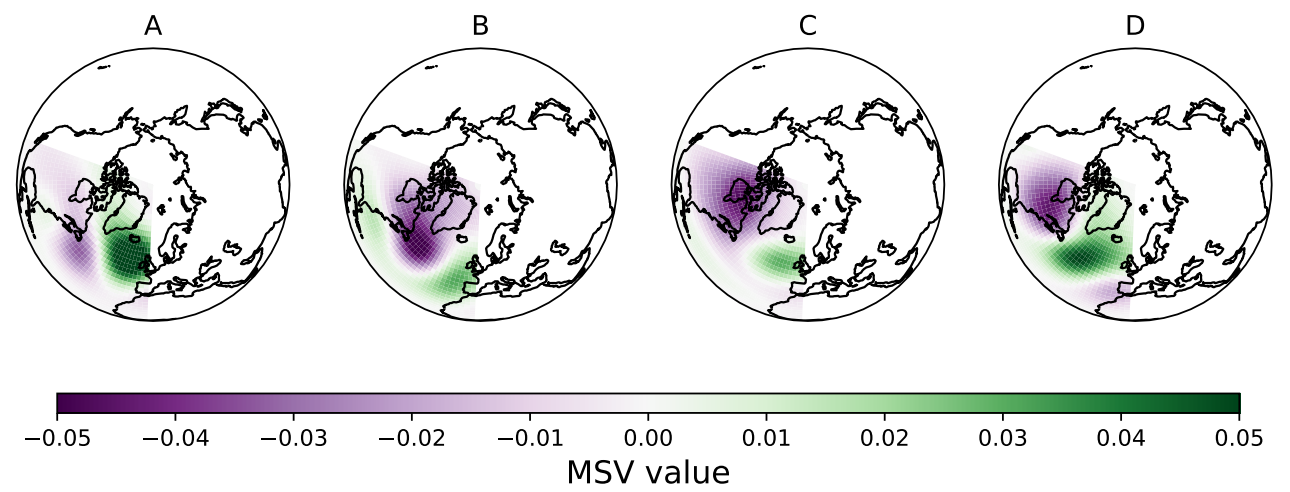

FIG. 6. Physical projections of unstable MSVs (computed for $M=3$ ) at transitions associated with persistent states (i.e. residency greater than 4 days in the state before and after the transition). The labels for each transition correspond to those discussed in TABLE 5. All projections use the same color bar scale. As the MSVs and EOFs are unit normalized and the EOFs are orthogonal, the projections shown here are also unit normalized. 

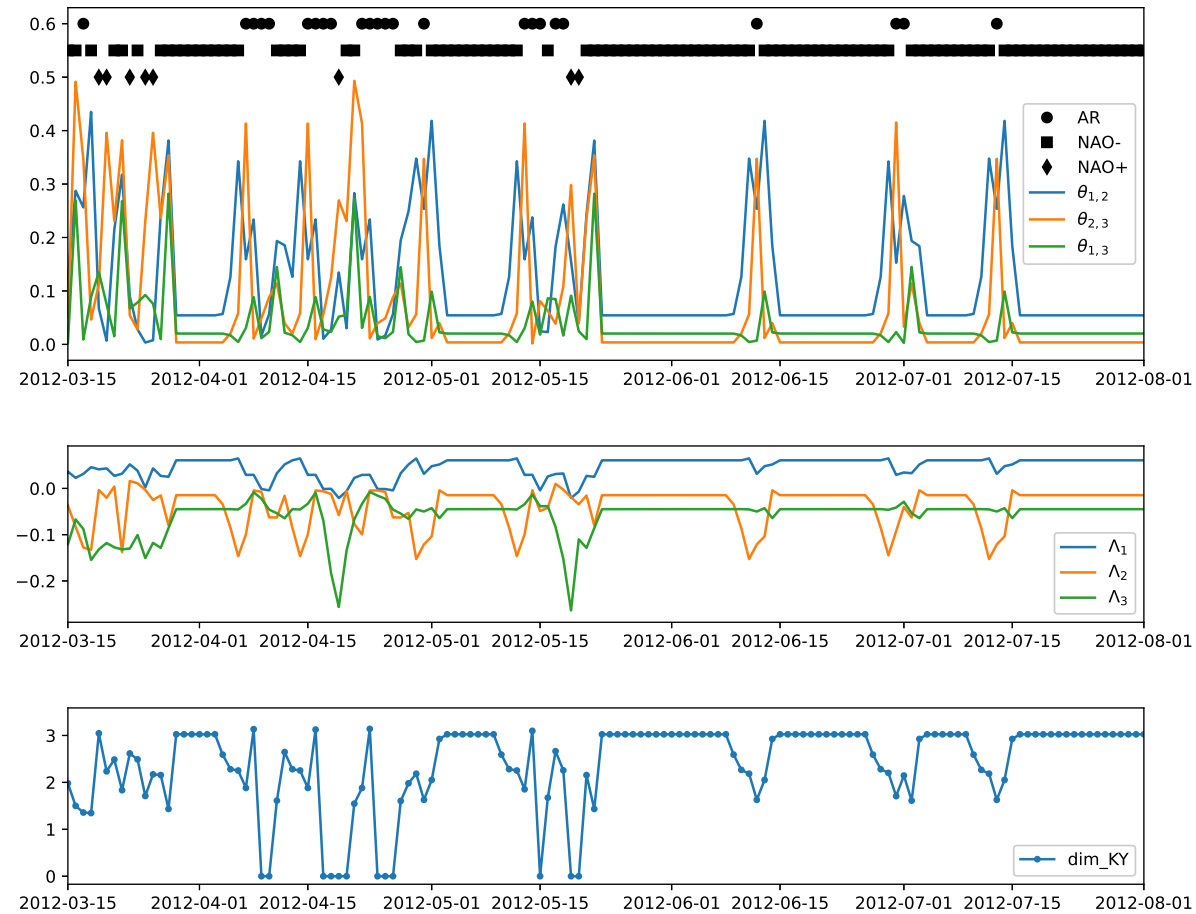

(a)
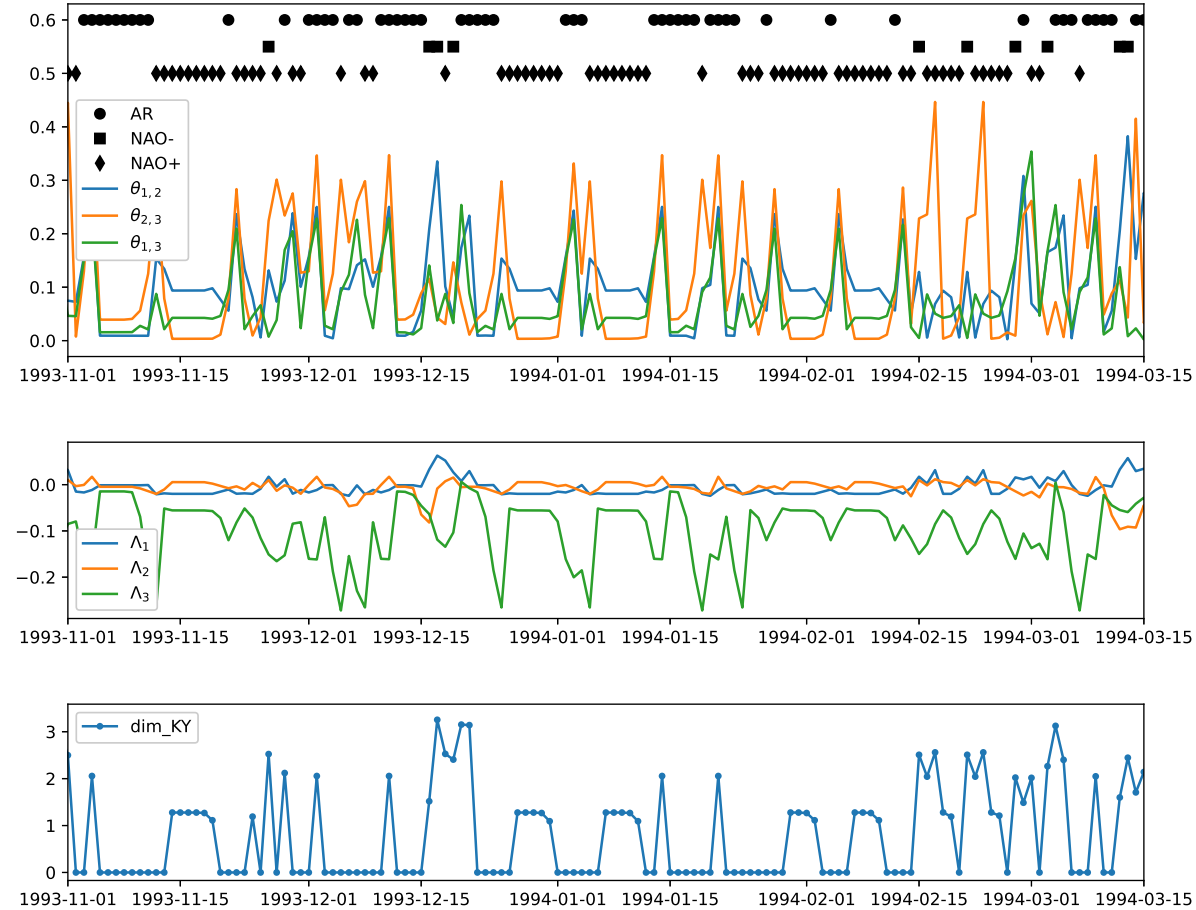

(b)

FIG. 7. Transient behavior of the leading CLV alignments $\left(\theta_{1,2}, \theta_{2,3}\right.$, and $\left.\theta_{1,3}\right)$, growth rates $\left(\Lambda_{1}, \Lambda_{2}\right.$, and $\left.\Lambda_{3}\right)$, and finite-time dimension for two different but representative time segments using push forward $M=3$. We also plot the state indicators to compare to transitions. 


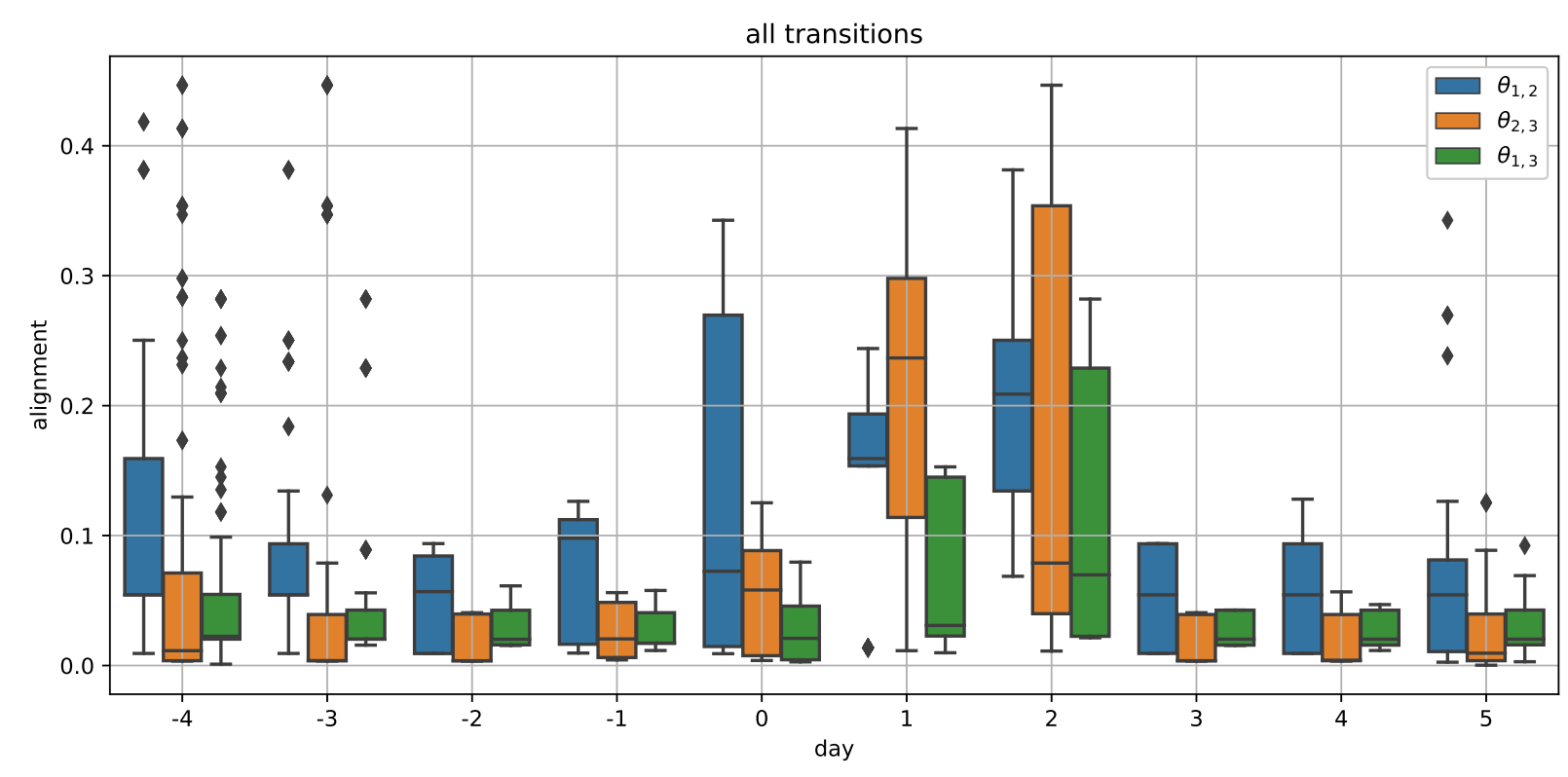

FIG. 8. Box and whisker plots of $\theta_{1,2}, \theta_{2,3}$, and $\theta_{1,3}$ around each transition with Day 0 indicating the last day in the previous state and Day 1 the first day in the following state. Diamonds indicate outlier values. The transitions have been filtered to only include those associated with residencies longer than 4 days both before and after the 1052 transition. 


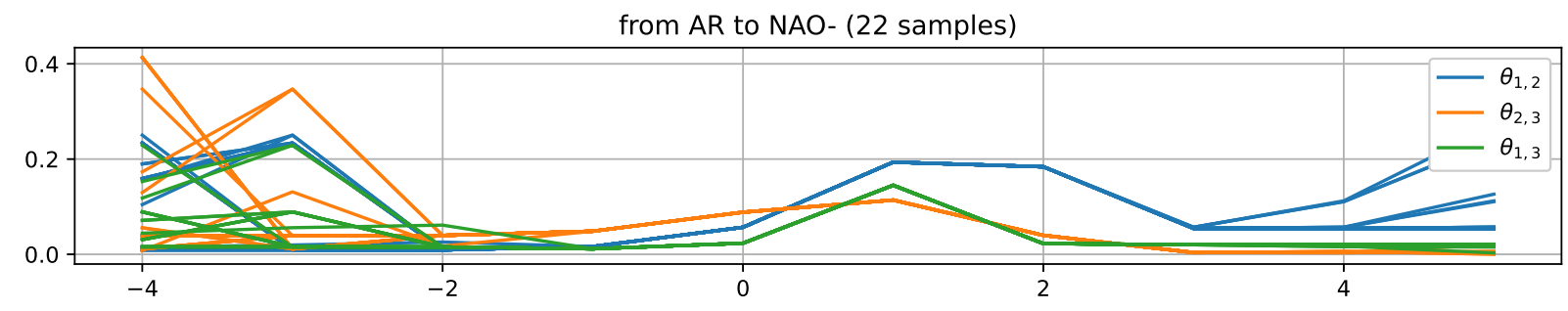

from AR to $\mathrm{NAO}+(21$ samples)

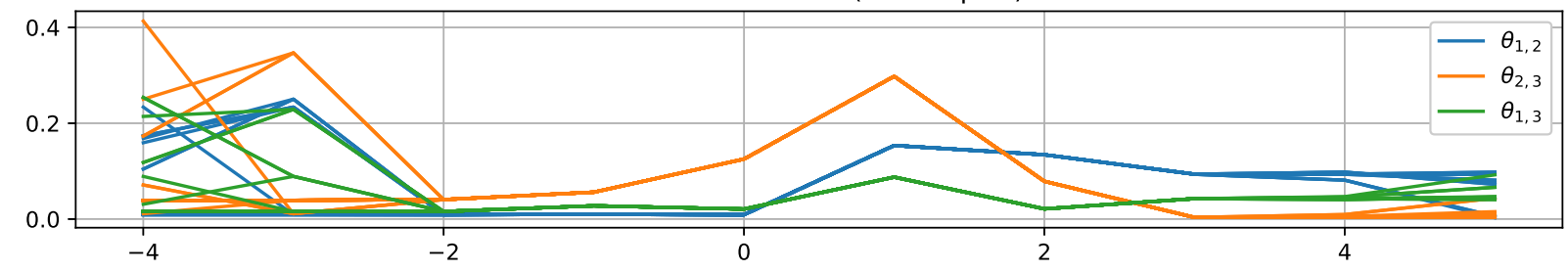

from NAO- to $\mathrm{NAO}+(26$ samples)

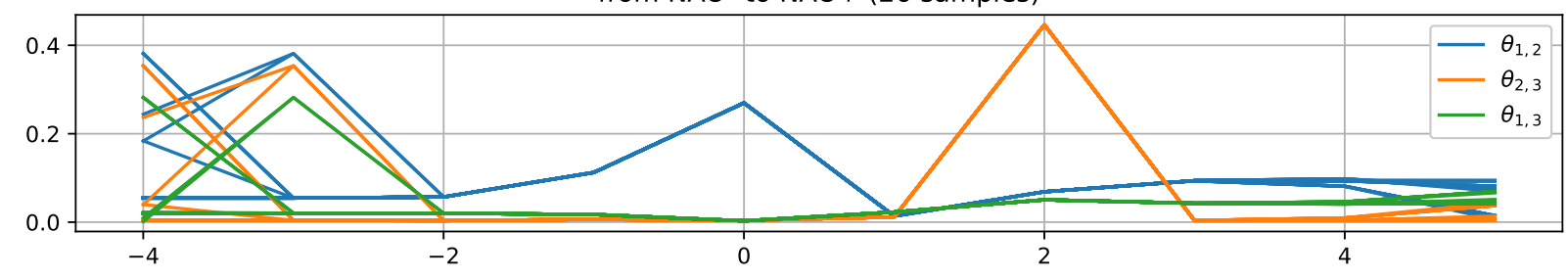

from NAO- to AR (33 samples)

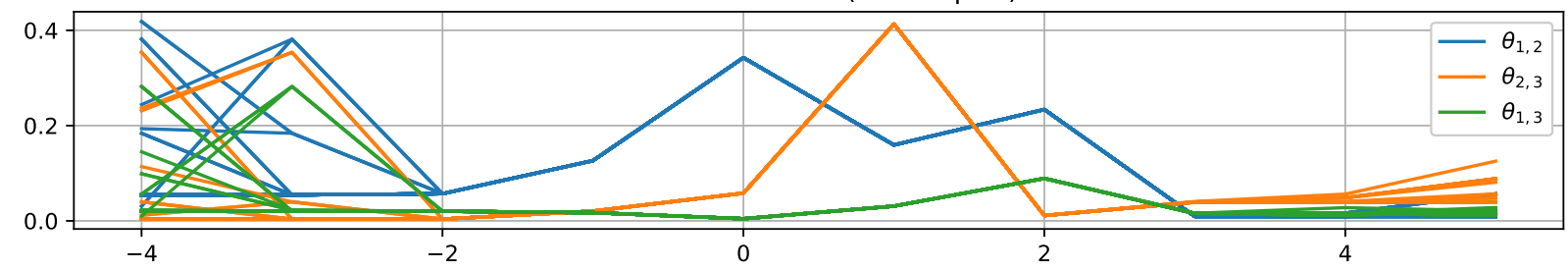

from NAO+ to NAO- (21 samples)
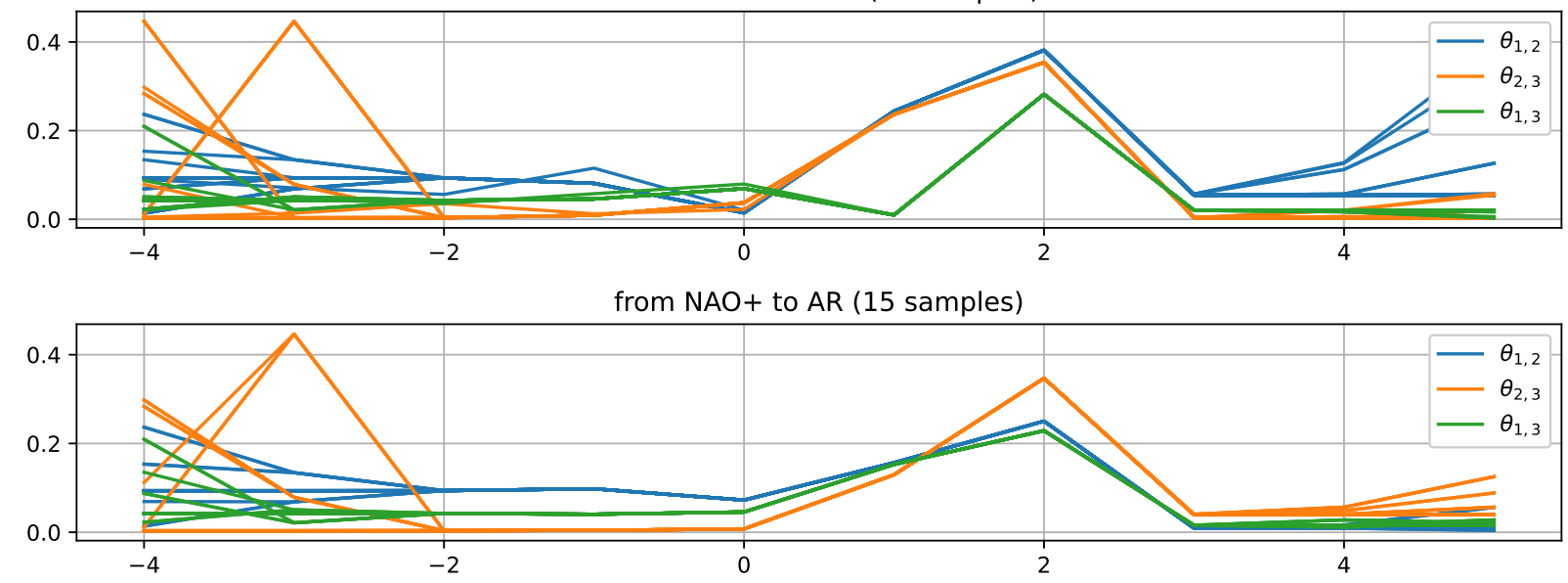

FIG. 9. Collective trajectories of $\theta_{1,2}, \theta_{2,3}$, and $\theta_{1,3}$ separated by specific transition. The transitions have been filtered as in FIG. 8. 

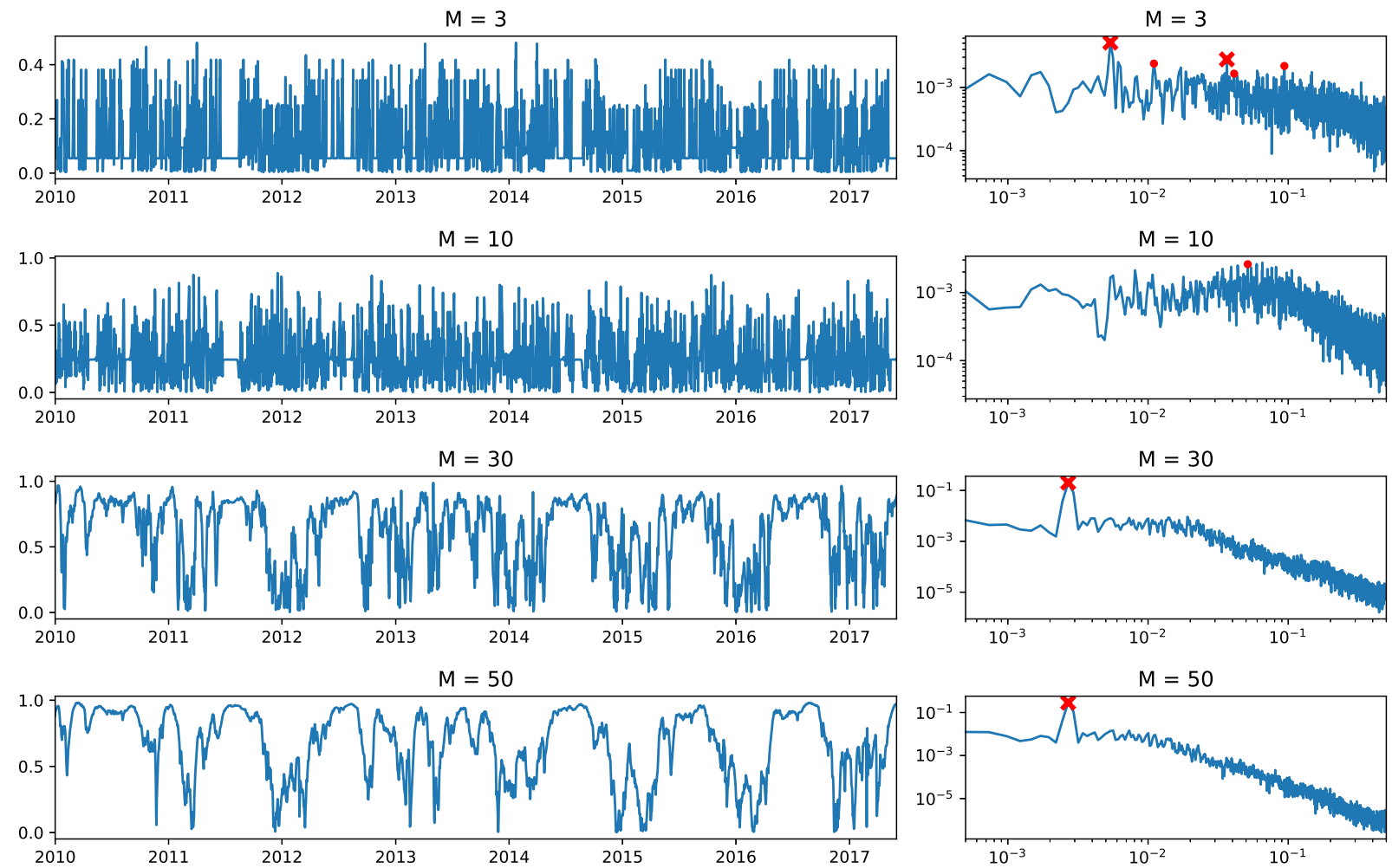

(a)

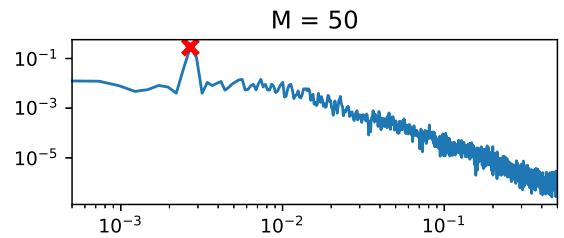

(b)

FIg. 10. (a) Alignment of the leading two CLVs for different push forward steps. From top to bottom: raw $\mathrm{NAO}^{-}$signal, $M=3, M=10, M=30, M=50$. (b) Power spectral density of the corresponding alignment time series. Red dots (crosses) indicate peaks that are 2 (3) standard deviations away from neighboring measures. 


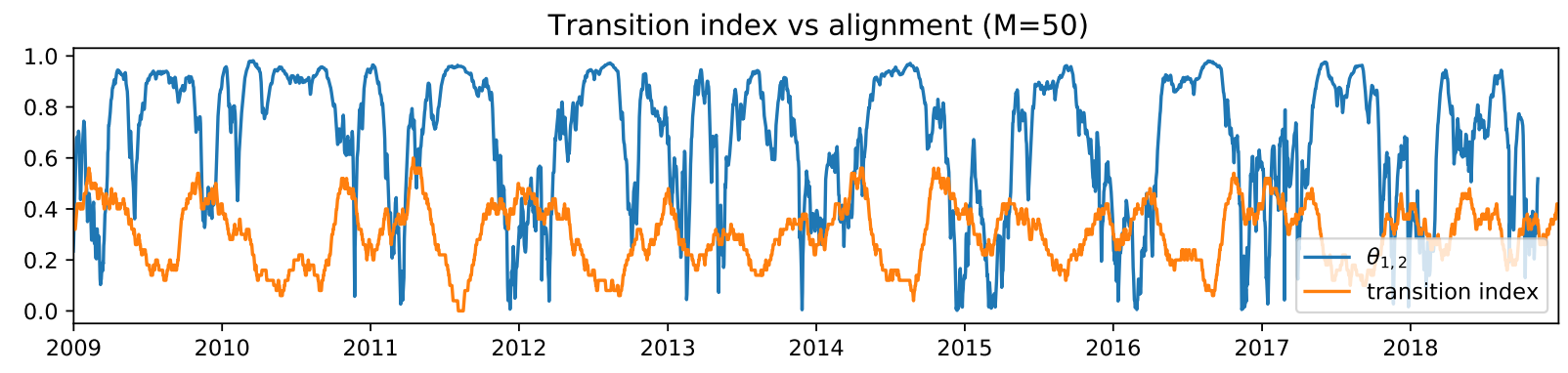

FIG. 11. Alignment of the leading two CLVs for push forward step $M=50$ compared to transition index 1059 calculated from Eq. (19). 

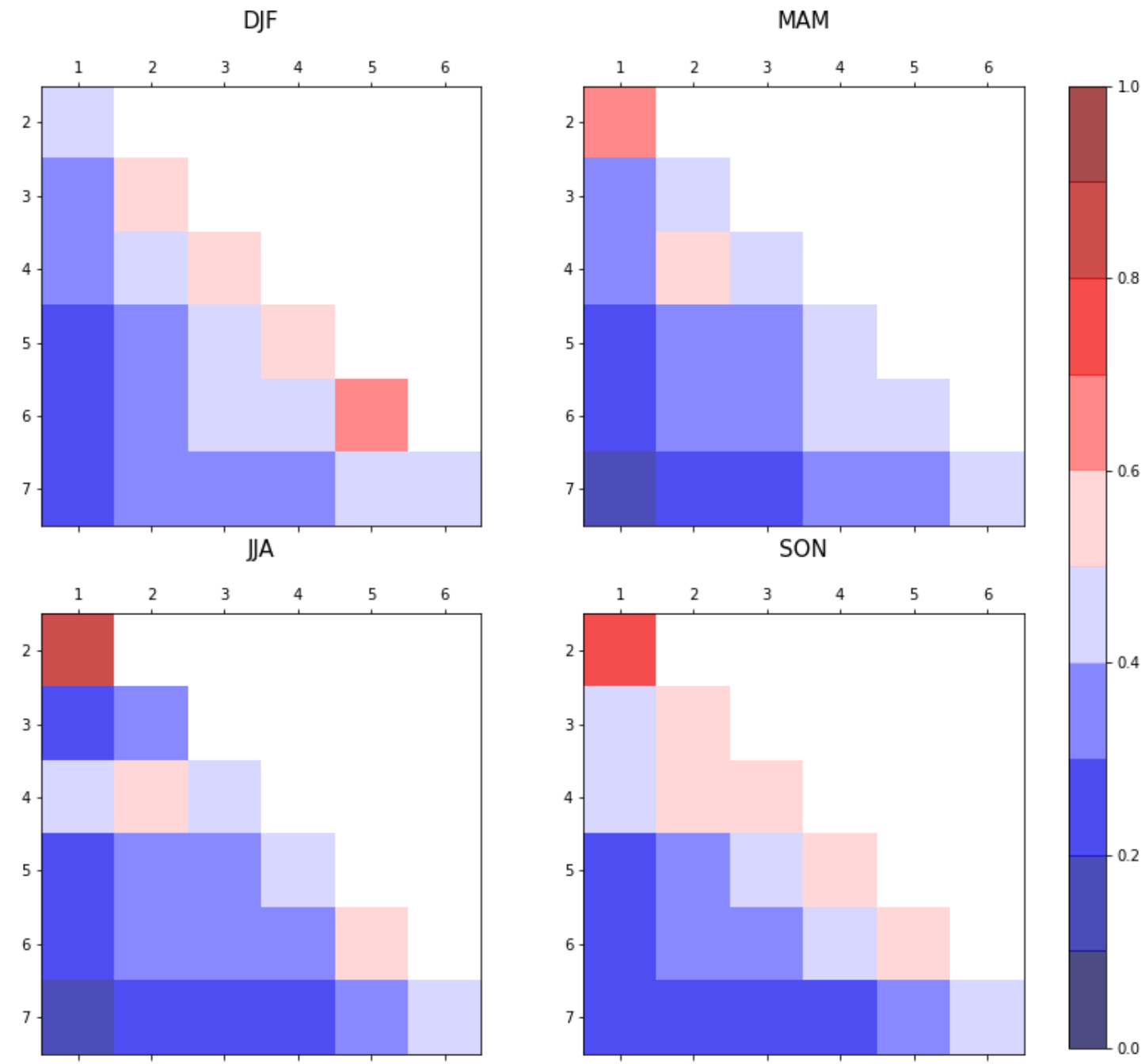

FIG. 12. Comparison of average alignment $\left(\overline{\theta_{i, j}}\right)$ of leading CLVs by season for push forward $M=50$. We see the strong alignment emerging in the JJA $\overline{\theta_{1,2}}$, and a weak alignment in both SON and DJF. Additionally we observe some seasonality in $\overline{\theta_{2,3}}$ and $\overline{\theta_{3,4}}$, with both peaking in DJF. 

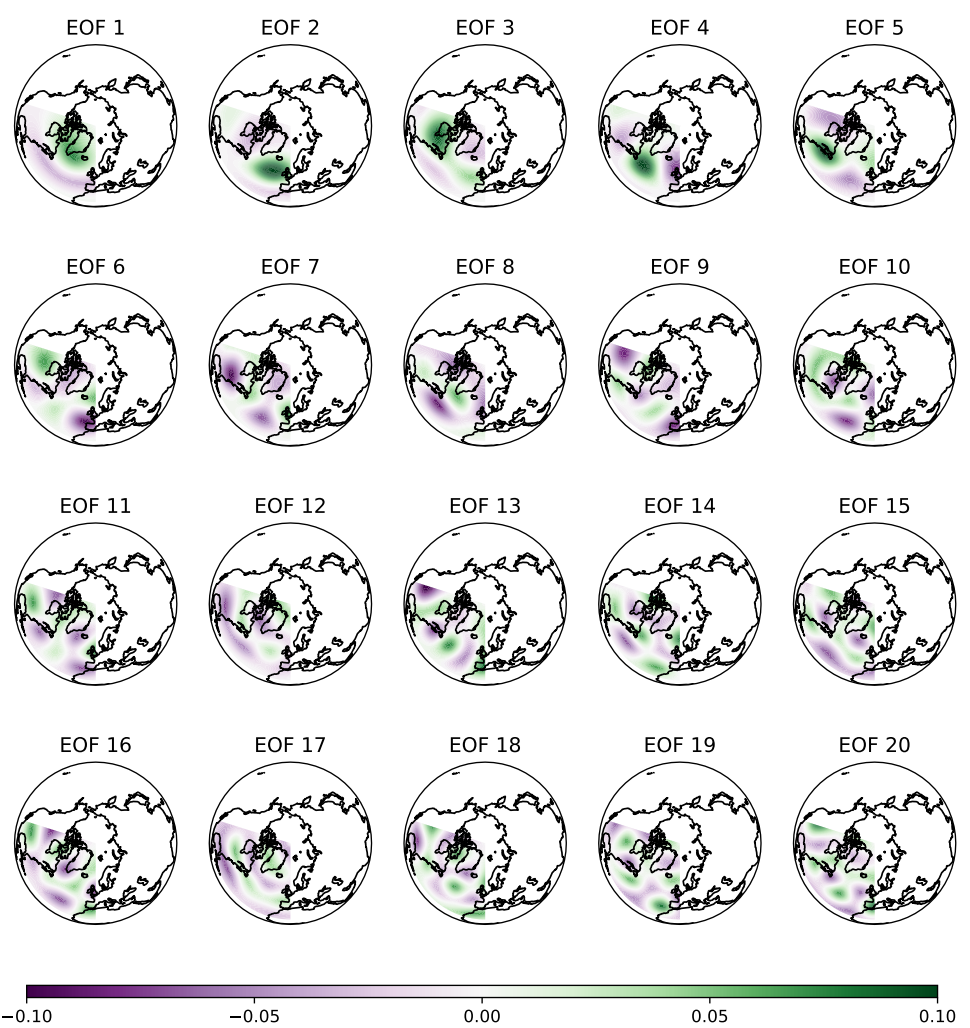

$-0.05$

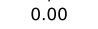

EOF value

Fig. A1. Leading 20 EOFs of daily $500 \mathrm{hPa}$ geopotential height anomalies in the North Atlantic sector

$\left(20^{\circ} \mathrm{N}-90^{\circ} \mathrm{N}\right.$ and $\left.110^{\circ} \mathrm{W}-0^{\circ} \mathrm{E}\right)$ of the daily NCEP/NCAR reanalysis data (Kalnay et al. 1996). All EOFs are unit normalized and use the same color scale shown at the bottom. 

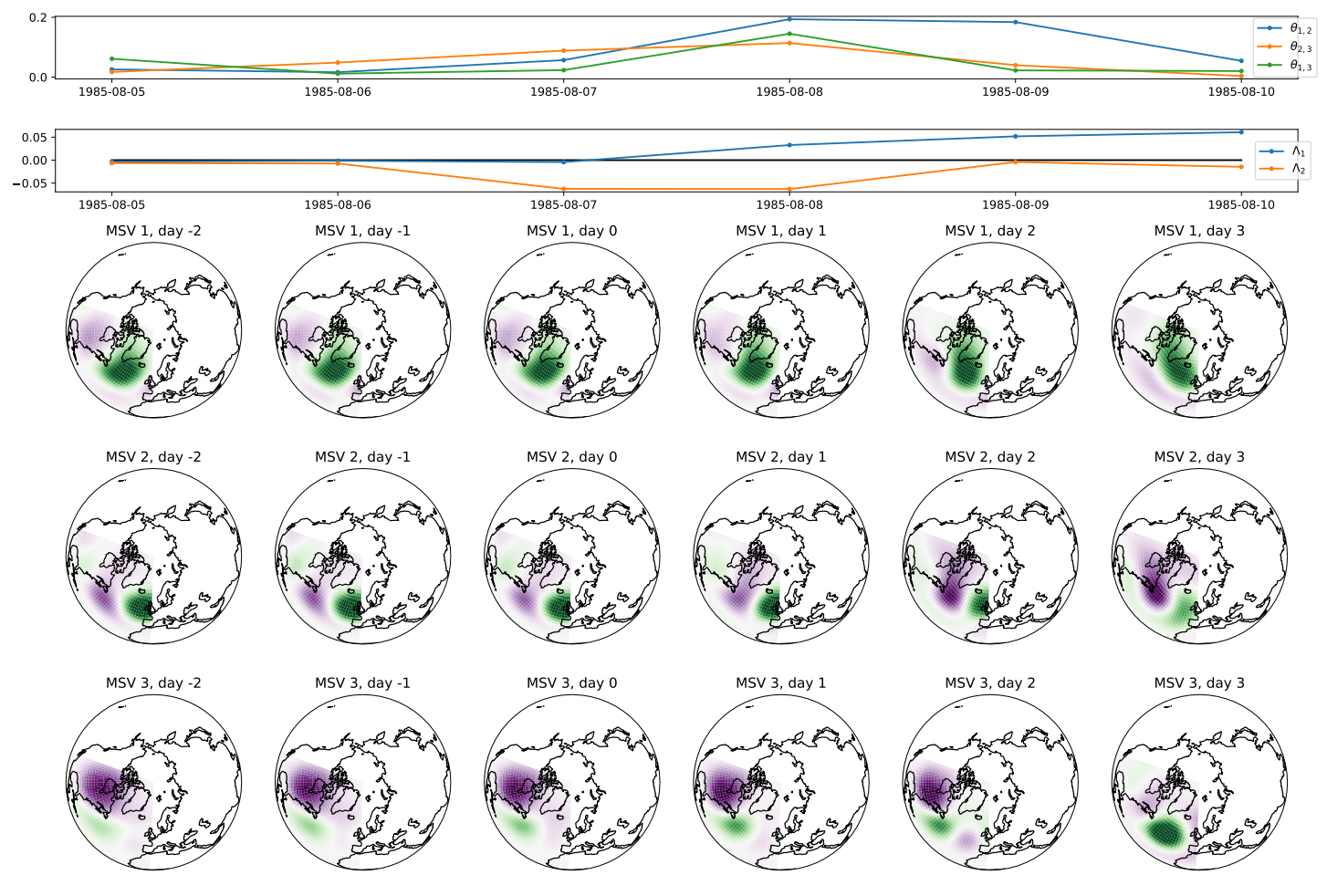

$-0.05$

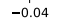

$-0.03$

MSV value

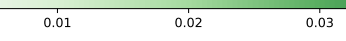

0.04

Fig. D1. Alignment, FTEs, and unit normalized physical projections of the leading MSVs throughout the transition from a persistent Atlantic Ridge state to a persistent negative NAO state. 

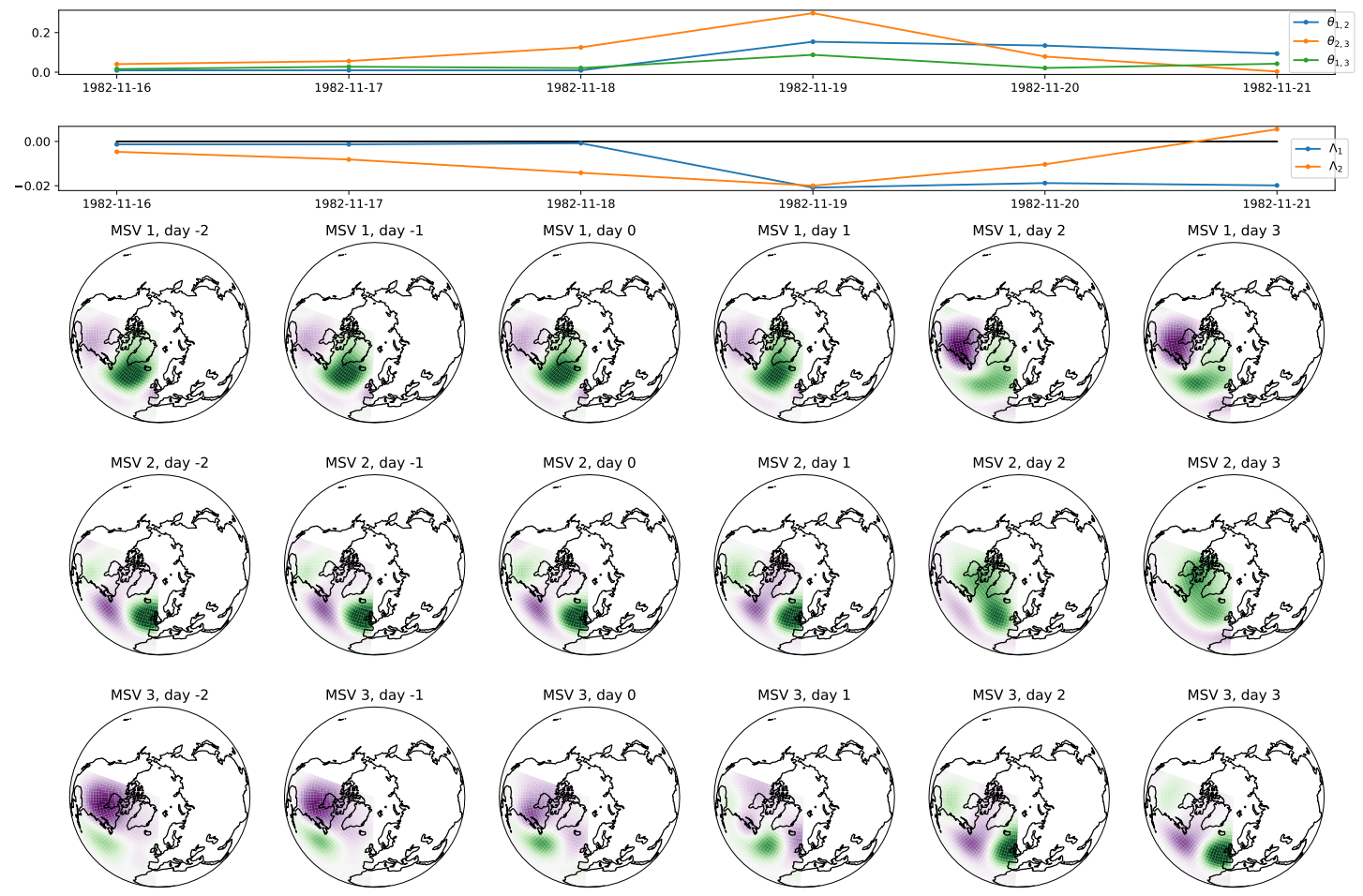

$-0.05$

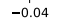

$-0.03$

0.00
MSV value

Fig. D2. Same as Fig. D1, but for the Atlantic Ridge to positive NAO transition. 

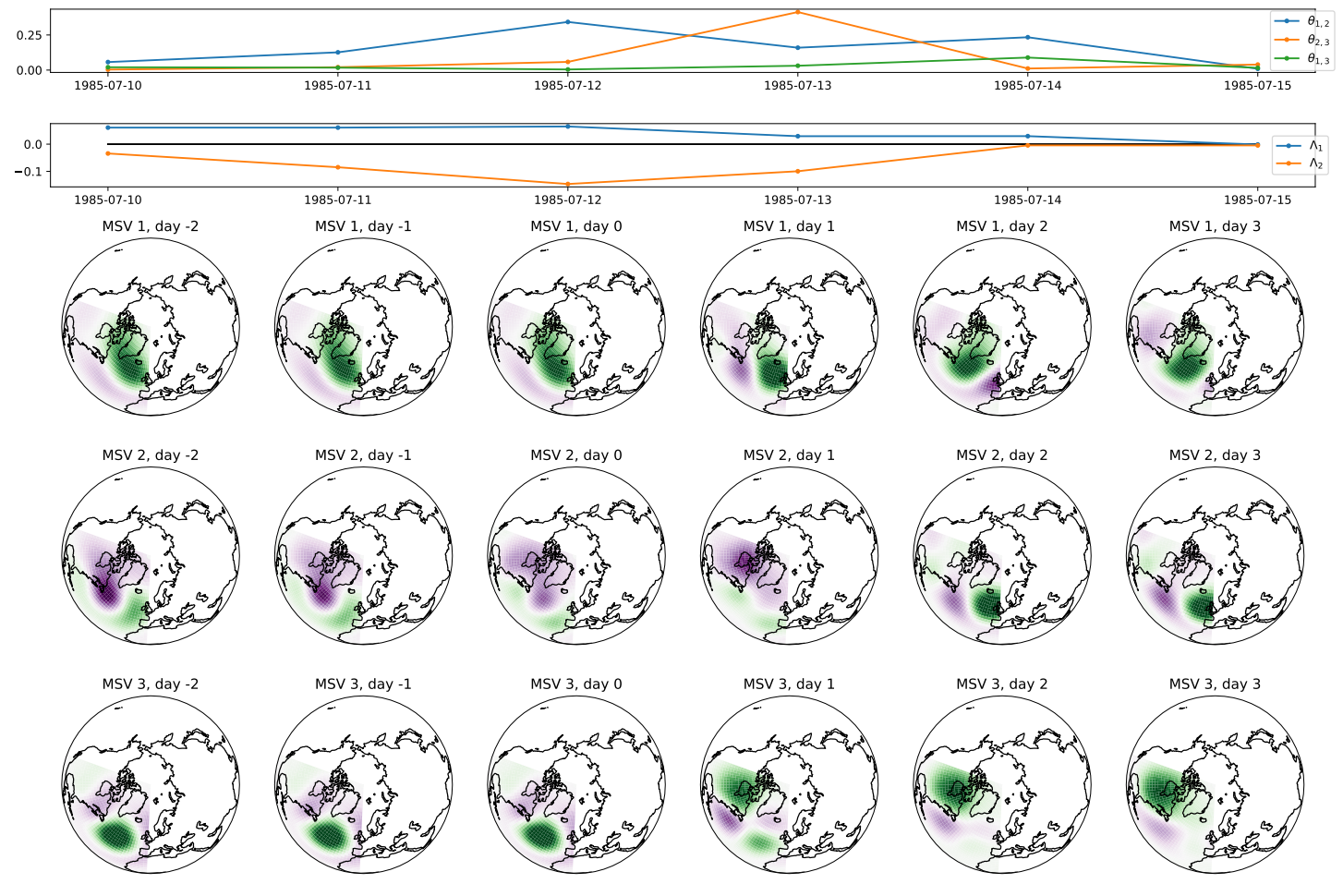

$-0.05$

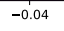

$-0.03$

0.00
MSV value

0.03

0.04

Fig. D3. Same as Fig. D1, but for the negative NAO to Atlantic Ridge transition. 

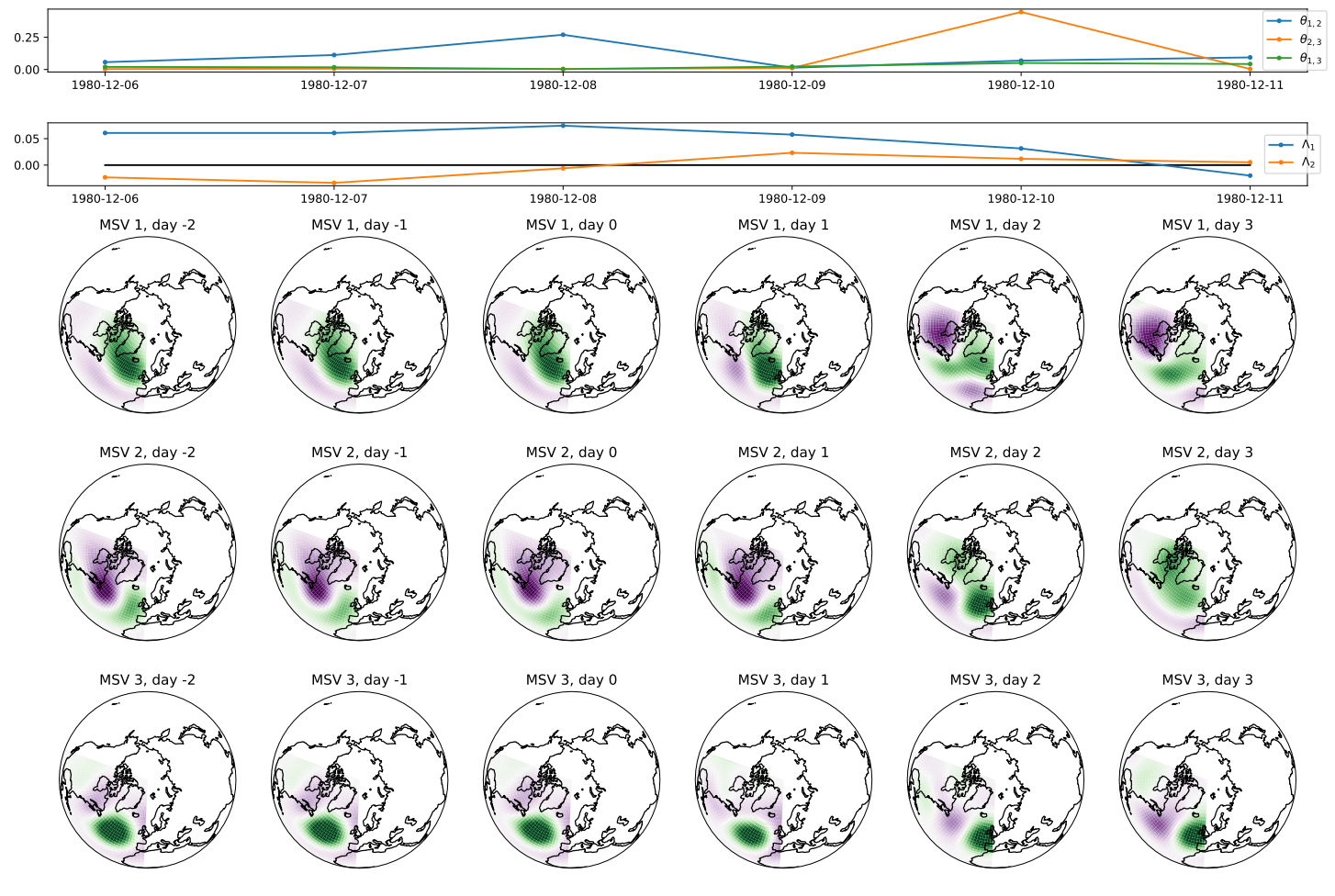

$-0.05$

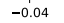

$-0.03$

MSV value

0.04

Fig. D4. Same as FIG. D1, but for the negative NAO to positive NAO transition. 

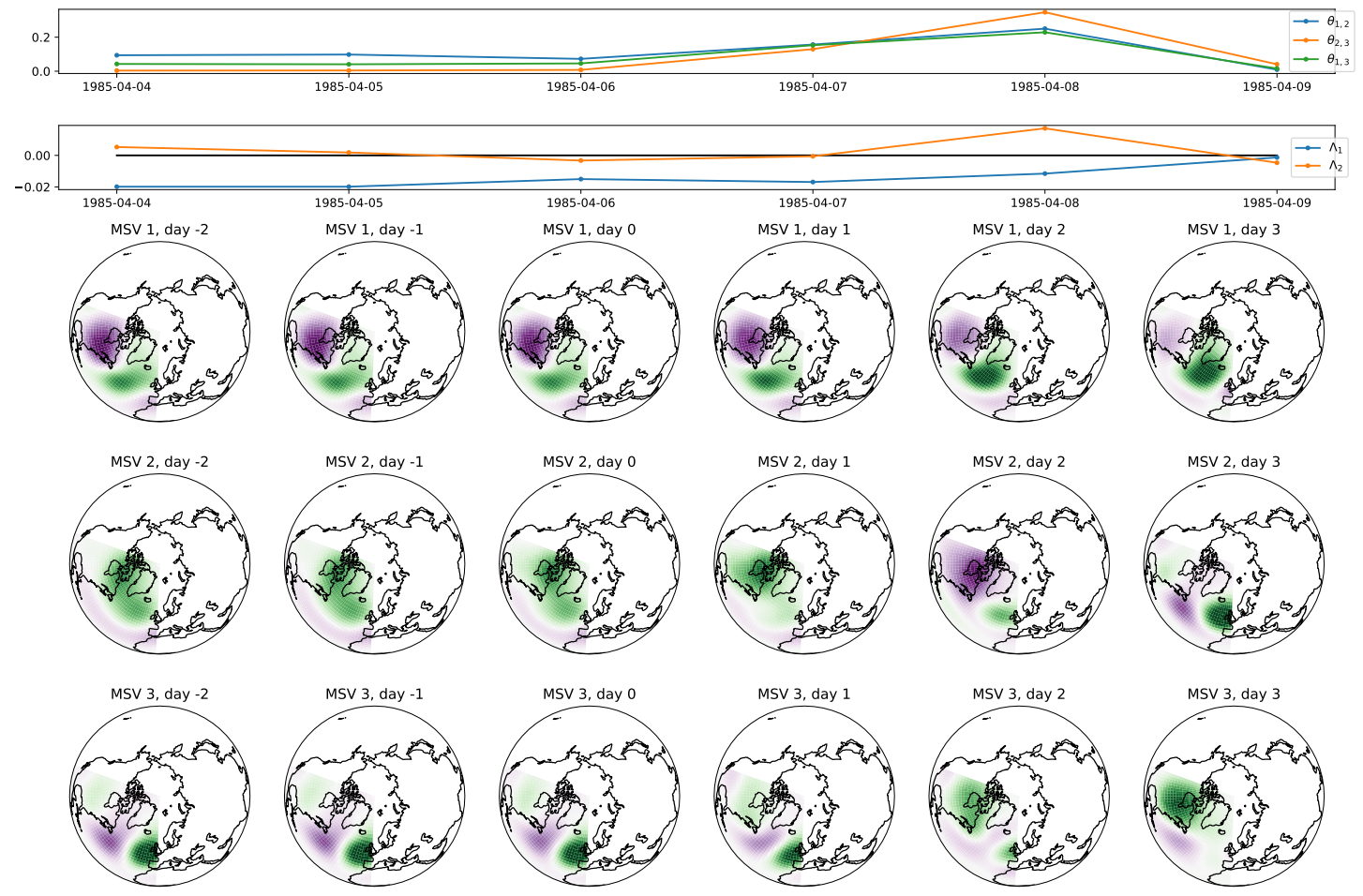

$-0.05$

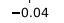

$-0.03$

0.00
MSV value

0.04

Fig. D5. Same as FIg. D1, but for the positive NAO to Atlantic Ridge transition. 

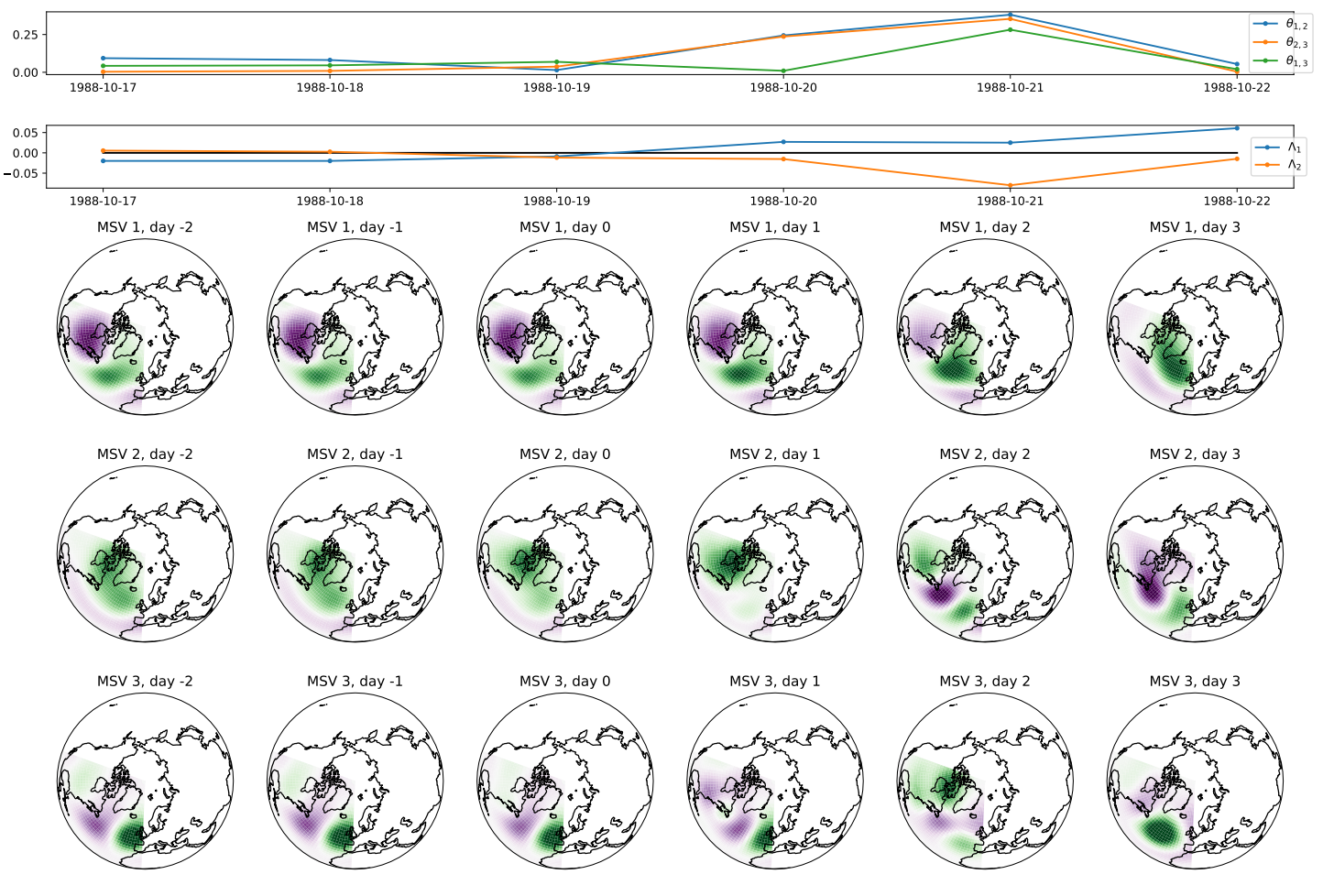

$-0.05$

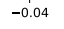

$-0.03$

MSV value

0.04

Fig. D6. Same as FIG. D1, but for the positive NAO to negative NAO transition. 